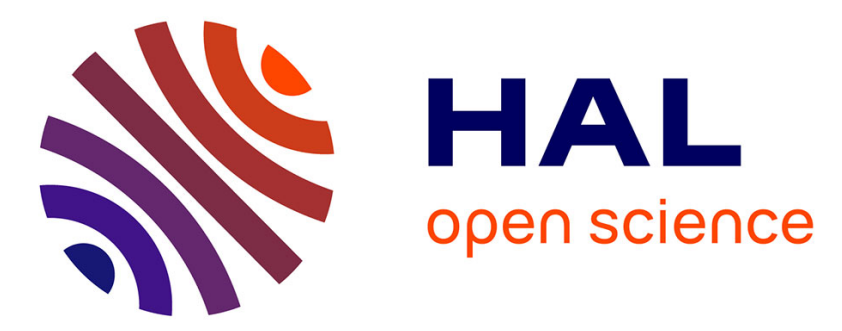

\title{
Surveying and comparing simultaneous sparse approximation (or group lasso) algorithms
}

\author{
Alain Rakotomamonjy
}

\section{To cite this version:}

Alain Rakotomamonjy. Surveying and comparing simultaneous sparse approximation (or group lasso) algorithms. 2010. hal-00328185v3

\section{HAL Id: hal-00328185 \\ https://hal.science/hal-00328185v3}

Preprint submitted on 8 Apr 2010

HAL is a multi-disciplinary open access archive for the deposit and dissemination of scientific research documents, whether they are published or not. The documents may come from teaching and research institutions in France or abroad, or from public or private research centers.
L'archive ouverte pluridisciplinaire HAL, est destinée au dépôt et à la diffusion de documents scientifiques de niveau recherche, publiés ou non, émanant des établissements d'enseignement et de recherche français ou étrangers, des laboratoires publics ou privés. 


\title{
Surveying and comparing simultaneous sparse approximation (or group-lasso) algorithms
}

\author{
A. Rakotomamonjy ${ }^{1, *}$ \\ LITIS EA4108, University of Rouen
}

\begin{abstract}
In this paper, we survey and compare different algorithms that, given an overcomplete dictionary of elementary functions, solve the problem of simultaneous sparse signal approximation, with common sparsity profile induced by a $\ell_{p}-\ell_{q}$ mixed-norm. Such a problem is also known in the statistical learning community as the group lasso problem. We have gathered and detailed different algorithmic results concerning these two equivalent approximation problems. We have also enriched the discussion by providing relations between several algorithms. Experimental comparisons of several of the detailed algorithms have also been carried out. The main lesson learned from these experiments is that depending on the performance measure, greedy approaches and iterative reweighted algorithms are the efficient algorithms either in term of computational complexities or in term of sparsity recovery.

Keywords: simultaneous sparse approximation, block sparse regression, group lasso, iterative reweighted algorithms
\end{abstract}

\section{Introduction}

Since several years now, there has been a lot of interest about sparse signal approximation. This large interest comes from frequent wishes of practitioners

\footnotetext{
${ }^{*}$ Corresponding author

Email address: alain.rakotomamonjy@insa-rouen.fr (A. Rakotomamonjy )

${ }^{1}$ Complete postal address : LITIS EA4108, University of Rouen, Avenue de l'université, 76800 Saint Etienne du Rouvray, France.
}

Preprint submitted to Elsevier

April 8, 2010 
to represent data in the most parsimonious way.

Recently, researchers have focused their efforts on a natural extension of sparse approximation problem which is the problem of finding jointly sparse representations of multiple signal vectors. This problem is also known as simultaneous sparse approximation and it can be stated as follows. Suppose we have several signals describing the same phenomenon, and each signal is contaminated by noise. We want to find the sparsest approximation of each signal by using the same set of elementary functions. Hence, the problem consists in finding the best approximation of each signal while controlling the number of functions involved in all the approximations.

Such a situation arises in many different application domains such as sensor networks signal processing [28, 11], neuroelectromagnetic imaging 21, 35, 53], source localization [29], image restoration [17], and distributed compressed sensing 25$]$.

\subsection{Problem formalization}

Formally, the problem of simultaneous sparse approximation is the following. Suppose that we have measured $L$ signals $\left\{\mathbf{s}_{i}\right\}_{i=1}^{L}$ where each signal is of the form $\mathbf{s}_{i}=\boldsymbol{\Phi} \mathbf{c}_{i}+\epsilon$ where $\mathbf{s}_{i} \in \mathbb{R}^{N}, \boldsymbol{\Phi} \in \mathbb{R}^{N \times M}$ is a matrix of unit-norm elementary functions, $\mathbf{c}_{i} \in \mathbb{R}^{M}$ a weighting vector and $\epsilon$ is a noise vector. $\boldsymbol{\Phi}$ will be denoted in the sequel as the dictionary matrix. Since we have several signals, the overall measurements can be written as :

$$
\mathbf{S}=\mathbf{\Phi} \mathbf{C} \mathcal{E}
$$

with $\mathbf{S}=\left[\begin{array}{llll}\mathbf{s}_{1} & \mathbf{s}_{2} & \cdots & \mathbf{s}_{L}\end{array}\right]$ a signal matrix, $\mathbf{C}=\left[\begin{array}{llll}\mathbf{c}_{1} & \mathbf{c}_{2} & \cdots & \mathbf{c}_{L}\end{array}\right]$ a coefficient matrix and $\mathcal{E}$ a noise matrix. Note that in the sequel, we have adopted the following notations : $c_{i,}$. and $c_{\cdot, j}$ respectively denote the $i$ th row and $j$ th column of matrix $\mathbf{C}$ and $c_{i, j}$ is the $i$ th element in the $j$ th column of $\mathbf{C}$.

For the simultaneous sparse approximation (SSA) problem, the goal is then to recover the matrix $\mathbf{C}$ given the signal matrix $\mathbf{S}$ and the dictionary $\mathbf{\Phi}$ under the hypothesis that all signals $\mathbf{s}_{i}$ share the same sparsity profile. This latter 
hypothesis can also be translated into the coefficient matrix $\mathbf{C}$ having a minimal number of non-zero rows. In order to measure the number of non-zero rows of $\mathbf{C}$, a possible criterion is the so-called row-support or row-diversity measure of a coefficient matrix defined as

$$
\operatorname{rowsupp}(\mathbf{C})=\left\{i \in[1 \cdots M]: c_{i, k} \neq 0 \text { for some } k\right\}
$$

The row-support of $\mathbf{C}$ tells us which atoms of the dictionary have been used for building the signal matrix. Hence, if the cardinality of the row-support is lower than the dictionary cardinality, it means that at least one atom of the dictionary has not been used for synthesizing the signal matrix. Then, the row- $\ell_{0}$ pseudonorm of a coefficient matrix can be defined as : $\|\mathbf{C}\|_{\text {row-0 }}=|\operatorname{rowsupp}(\mathbf{C})|$. According to this definition, the simultaneous sparse approximation problem can be stated as

$$
\begin{array}{ll}
\min _{\mathbf{C}} & \frac{1}{2}\|\mathbf{S}-\mathbf{\Phi} \mathbf{C}\|_{F}^{2} \\
\text { st. } & \|\mathbf{C}\|_{\text {row }-0} \leq T
\end{array}
$$

where $\|\cdot\|_{F}$ is the Frobenius norm and $T$ a user-defined parameter that controls the sparsity of the solution. Note that the problem can also take the different form :

$$
\begin{array}{ll}
\min _{\mathbf{C}} & \|\mathbf{C}\|_{\text {row }-0} \\
\text { st. } & \frac{1}{2}\|\mathbf{S}-\mathbf{\Phi} \mathbf{C}\|_{F} \leq \epsilon
\end{array}
$$

For this latter formulation, the problem translates in minimizing the number of non-zero rows in the coefficient matrix $\mathbf{C}$ while keeping control on the approximation error. Both problems (21) and (3) are appealing for their formulation clarity. However, similarly to the single signal approximation case, solving these optimization problems are notably intractable because $\|\cdot\|_{\text {row-0 }}$ is a discretevalued function. Two ways of addressing these intractable problems (2) and (3) are possible : relaxing the problem by replacing the $\|\cdot\|_{\text {row }-0}$ function with a more tractable row-diversity measure or by using some suboptimal algorithms.

A large class of relaxed versions of $\|\cdot\|_{\text {row }-0}$ proposed in the literature are 
encompassed into the following form :

$$
J_{p, q}(\mathbf{C})=\sum_{i}\left\|c_{i, \cdot}\right\|_{q}^{p} \quad \text { with }\left\|c_{i, \cdot}\right\|_{q}=\left(\sum_{j}\left|c_{i, j}\right|^{q}\right)^{1 / q}
$$

where typically $p \leq 1$ and $q \geq 1$. This penalty term can be interpreted as the $\ell_{p}$ quasi-norm of the sequence $\left\{\left\|c_{i,}\right\|_{q}\right\}_{i}$. Note that as $p$ converges to $0, J_{p, q}(\mathbf{C})$ provably converges towards $\sum_{i} \log \left(\left\|c_{i,},\right\|\right)$. According to this relaxed version of the row-diversity measure, most of the algorithms proposed in the literature try to solve the relaxed problem :

$$
\min _{\mathbf{C}} \frac{1}{2}\|\mathbf{S}-\mathbf{\Phi} \mathbf{C}\|_{F}^{2}+\lambda J_{p, q}(\mathbf{C})
$$

where $\lambda$ is another user-defined parameter that balances the approximation error and the sparsity-inducing penalty $J_{p, q}(\mathbf{C})$. The choice of $p$ and $q$ results in a compromise between the row-support sparsity and the convexity of the optimization problem. Indeed, problem (4) is known to be convex when $p, q \geq 1$ while it is known to produce a row-sparse matrix $\mathbf{C}$ if $p \leq 1$ (due to the penalty function singularity at $\mathbf{C}=0[15])$.

The simultaneous sparse approximation problem as described here is equivalent to several other problems studied in other research communities. Problem (4) can be reformulated so as to make clear its relation with some other problems denoted as the $\ell_{p}-\ell_{q}$ group lasso [55] or block-sparse regression [26] in statistics or block-sparse signal recovery 13] in signal processing. Indeed, let us define :

$$
\tilde{\mathbf{s}}=\left(\begin{array}{c}
\mathbf{s}_{1} \\
\vdots \\
\mathbf{s}_{L}
\end{array}\right) \quad \tilde{\boldsymbol{\Phi}}=\left(\begin{array}{ccc}
\mathbf{\Phi} & & (0) \\
& \ddots & \\
(0) & & \mathbf{\Phi}
\end{array}\right) \quad \tilde{\mathbf{c}}=\left(\begin{array}{c}
\mathbf{c}_{1} \\
\vdots \\
\mathbf{c}_{L}
\end{array}\right)
$$

then, problem (44) can be equivalently rewritten as :

$$
\min _{\tilde{\mathbf{c}}} \frac{1}{2}\|\tilde{\mathbf{s}}-\tilde{\mathbf{\Phi}} \tilde{\mathbf{c}}\|_{2}^{2}+\lambda J_{p, q}^{\prime}(\tilde{\mathbf{c}})
$$

where $J_{p, q}^{\prime}(\tilde{\mathbf{c}})=\sum_{i=1}^{M}\left\|\tilde{\mathbf{c}}_{g_{i}}\right\|_{q}^{p}$ with $g_{i}$ being all the indices in $\tilde{\mathbf{c}}$ related to the $i$-th element of the dictionary matrix $\mathbf{\Phi}$. 
As we have already stated, simultaneous sparse approximation and equivalent problems have been investigated by diverse communities and have also been applied to various application problems. Since the literature on these topics has been overwhelming since the last few years, The aim of this work is to gather results from different communities (statistics, signal processing and machine learning) so as to survey, analyze and compare different proposed algorithms for solving optimization problem (4) for different values of $p$ and $q$. In particular, instead of merely summarizing existing results, we enrich our survey by providing results like proof of convergence, formal relations between different algorithms and experimental comparisons that were not available in the literature. These experimental comparisons essentially focus on the computational complexity of the different methods, on their ability of recovering the signal sparsity pattern and on the quality of approximation they provided evaluated through mean-square error.

Note that recently, there has been various works which addressed the simultaneous sparse approximation in the noiseless case [44, 42] and many works which aims at providing theoretical approximation guarantees in both noiseless and noisy cases 66, 13, 31, 14, 23, 27]. Surveying these works is beyond the scope of this paper and we suggest interested readers to follow for instance these pointers and references therein.

The most frequent case of problem (4) encountered in the literature is the one where $p=1$ and $q=2$. This case is the simpler one since it leads to a convex problem. We discuss different algorithms for solving this problem in Section 2 Then, we survey in Section 3 generic algorithms that are able to solve our approximation problems with different values of $p$ and $q$. These algorithms are essentially iterative reweighted $\ell_{1}$ or $\ell_{2}$ algorithms. Many works in the literature have focused on one algorithm for solving a particular case of problem (4). These specific algorithms are discussed in section 4 Notably, we present some greedy algorithms and discuss a sparse bayesian learning algorithm. The experimental comparisons we carried out aim at clarifying how each algorithm behaves in terms of computational complexity, in sparsity recovery and in approximation 
mean-square error. These results are described in Section 5 For a sake of reproducibility, the code used in this paper has been made freely available 2 . Final discussions and conclusions are given in Section 6 ]

\section{Solving the $\ell_{1}-\ell_{2}$ optimization problem}

When $p=1, q=2$, optimization problem (4) becomes a particular problem named as M-BP for Multiple Basis Pursuit in the sequel. It is a special case that deserves attention. Indeed, it seems to be the most natural extension of the socalled Lasso problem [45] or Basis Pursuit Denoising 7], since for $L=1$, it can be easily shown that problem (4) reduced to these two problems. The key point of this case is that it yields to a convex optimization problem and thus it can benefit from all properties resulting from convexity e.g global minimum. One of the first algorithm addressing such the M-BP problem is the one proposed by Cotter et al. 8] known as M-FOCUSS. Such an algorithm based on factored gradient descent works for any $p \leq 1$ and has been proved to converge towards a local or global (when $p=1$ ) minimum of problem (4) if it does not get stuck into a fixed point. Because, M-FOCUSS is better tailored for situations where $p \leq 1$, we post-pone its description to next section. The two algorithms on which we focus on herein, are based on a block-coordinate descent as proposed by Yuan et al. 55] for the group lasso and the one based on Landweber iterations of Fornasier et al. [17]. We have biased our survey towards these two approaches because of their efficiencies and their convergence properties.

\subsection{Block Coordinate descent}

The specific structure of the optimization problem (4) for $p=1$ and $q=2$ leads to a very simple block coordinate descent algorithm that we name as M-BCD. Here, we provide a more detailed derivation of the algorithm and we also give results that were not available in the Yuan et al. work [55]. We

\footnotetext{
${ }^{2}$ http://asi.insa-rouen.fr/enseignants/ arakotom/code/SSAindex.html
} 
provide a proof of convergence of the M-BCD algorithm and we show that if the dictionary is under-complete then it can be proved that the solution of the problem is equivalent to a simple shrinkage of the coefficient matrix.

\subsubsection{Deriving optimality conditions}

The M-BP optimization problem is the following

$$
\min _{\mathbf{C}} W(\mathbf{C})=\frac{1}{2}\|\mathbf{S}-\mathbf{\Phi} \mathbf{C}\|_{F}^{2}+\lambda \sum_{i}\left\|c_{i, .}\right\|_{2}
$$

where the objective function $W(\mathbf{C})$ is a non-smooth but convex function. Since the problem is unconstrained a necessary and sufficient condition for a matrix $\mathbf{C}^{\star}$ to be a minimizer of (6) is that $\mathbf{0} \in \partial W\left(\mathbf{C}^{\star}\right)$ where $\partial W(\mathbf{C})$ denotes the subdifferential of our objective value $W(\mathbf{C})$ 2]. By computing the subdifferential of $W(\mathbf{C})$ with respect to each row $c_{i}$, of $\mathbf{C}$, the KKT optimality condition of problem (6) is then

$$
-\mathbf{r}_{i}+\lambda g_{i, \cdot}=0 \quad \forall i
$$

where $\mathbf{r}_{i}=\phi_{i}^{t}(\mathbf{S}-\mathbf{\Phi C})$ and $g_{i}$, is the $i$-th row of a subdifferential matrix $\mathbf{G}$ of $J_{1,2}(\mathbf{C})=\sum_{i}\left\|c_{i,}\right\|_{2}$. According to the $J_{1,2}$ 's subdifferential definition given in the appendix, the KKT optimality conditions can be rewritten as

$$
\begin{aligned}
-\mathbf{r}_{i}+\lambda \frac{c_{i, \cdot}}{\left\|c_{i, \cdot}\right\|_{2}} & =\mathbf{0} \quad \forall i, \quad c_{i, \cdot} \neq \mathbf{0} \\
\left\|\mathbf{r}_{i}\right\|_{2} & \leq \lambda \quad \forall i, \quad c_{i, .}=\mathbf{0}
\end{aligned}
$$

A matrix $\mathbf{C}$ satisfying these equations can be obtained after the following algebra. Let us expand each $\mathbf{r}_{i}$ so that

$$
\begin{aligned}
\mathbf{r}_{i} & =\phi_{i}^{t}\left(\mathbf{S}-\mathbf{\Phi} \mathbf{C}_{-i}\right)-\phi_{i}^{t} \phi_{i} c_{i,} \\
& =T_{i}-c_{i}
\end{aligned}
$$

where $\mathbf{C}_{-i}$ is the matrix $\mathbf{C}$ with the $i$-th row being set to 0 and $T_{i}=\phi_{i}^{t}(\mathbf{S}-$ $\left.\mathbf{\Phi C}_{-i}\right)$. The second equality is obtained by remembering that $\phi_{i}^{t} \phi_{i}=1$. Then, equation (7) tells us that if $c_{i}$, is non-zero, $T_{i}$ and $c_{i}$. have to be collinear. Plugging all these points into equation (17) yields to an optimal solution that 
can be obtained as :

$$
c_{i, \cdot}=\left(1-\frac{\lambda}{\left\|T_{i}\right\|}\right)_{+} T_{i} \quad \forall i
$$

where $(x)_{+}=x$ if $x>0$ and 0 otherwise. From this update equation, we can derive a simple algorithm which consists in iteratively applying the update (9) to each row of $\mathbf{C}$.

\subsubsection{The algorithm and its convergence}

Our block-coordinate descent algorithm is detailed in Algorithm (11). It is a simple and efficient algorithm for solving M-BP.

Basically, the idea consists in solving each row $c_{i}$. at a time. By starting from a sparse solution like, $\mathbf{C}=0$, at each iteration, one checks for a given $i$

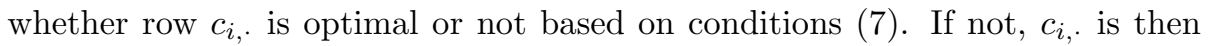
updated according to equation (9).

Although, such a block-coordinate algorithm does not converge in general for non-smooth optimization problem, Tseng [49] has shown that for an optimization problem which objective value is the sum of a smooth and convex function and a non-smooth but block-separable convex function, block-coordinate optimization converges towards the global minimum of the problem. Our proof of convergence is based on such properties and follows the same lines as the one proposed by Sardy et al. 39] for a single signal approximation.

Theorem 1. The $M-B C D$ algorithm converges to a solution of the M-Basis Pursuit problem given in Equation (6), where convergence is understood as any accumulation point of the $M-B C D$ algorithm is a minimum of problem (6) and the sequence of $\left\{\mathbf{C}_{k}\right\}$ generated by the algorithm is bounded.

Proof 1. Note that M-BP problem presents a particular structure with a smooth and differentiable convex function $\|\mathbf{S}-\mathbf{\Phi C}\|_{F}^{2}$ and a row-separable penalty function $\sum_{i} h_{i}\left(c_{i, .}\right)$ where $h(\cdot)$ is a continuous and convex function with respects to $c_{i, \cdot}$

Also note that our algorithm considers a cyclic rule where within each loop, for any $i \in[1, \cdots, M]$, each $c_{i,}$. is considered for optimization. The main partic- 


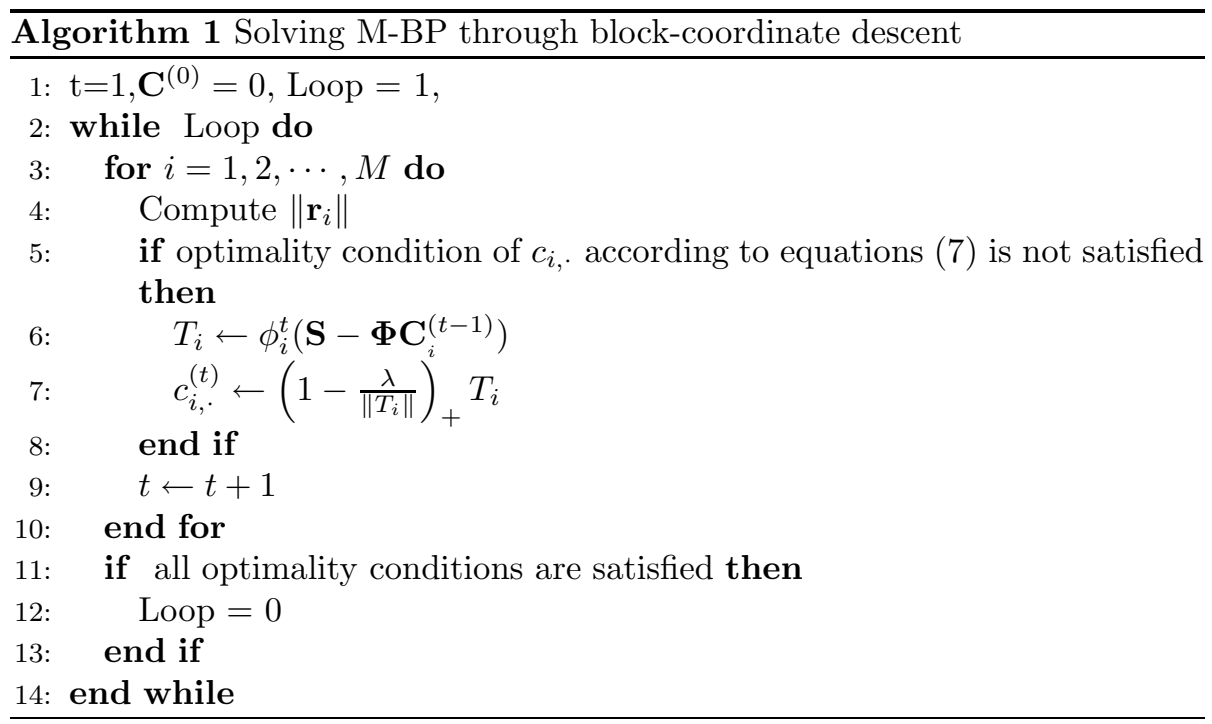

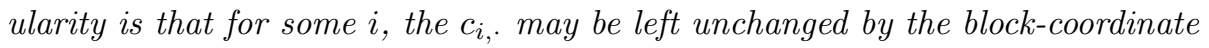
descent if already optimal. This occurs especially for row $c_{i, .}$ which are equal to 0 .

Then according to the special structure of the problem and the use of a cyclic rule, the results of Tseng [49] prove that our $M-B C D$ algorithm converges.

Another point we want to emphasize and that has not been shed to light yet is that this algorithm should be initialized at $\mathbf{C}=0$ so as to take advantage of the sparsity pattern of the solution. Indeed, by doing so, only few updates are needed before convergence.

Intuitively, we can understand this algorithm as an algorithm which tends to shrink to zero rows of the coefficient matrix that contribute poorly to the approximation. Indeed, $T_{i}$ can be interpreted as the correlation between the residual when row $i$ has been removed and $\phi_{i}$. Hence the smaller the norm of $T_{i}$ is, the less $\phi_{i}$ is relevant in the approximation. And according to equation (9), the smaller the resulting $c_{i,}$. is. Insight into this block-coordinate descent algorithm can be further obtained by supposing that $M \leq N$ and that $\boldsymbol{\Phi}$ is composed of orthonormal elements of $\mathbb{R}^{N}$, hence $\boldsymbol{\Phi}^{t} \boldsymbol{\Phi}=\mathbf{I}$. In such a situation, 
we have

$$
T_{i}=\phi_{i}^{t} \mathbf{S} \quad \text { and } \quad\left\|T_{i}\right\|_{2}^{2}=\sum_{k=1}^{L}\left(\phi_{i}^{t} s_{k}\right)^{2}
$$

and thus

$$
c_{i, \cdot}=\left(1-\frac{\lambda}{\sqrt{\sum_{k}^{L}\left(\phi_{i}^{t} s_{k}\right)^{2}}}\right)_{+} \phi_{i}^{t} \mathbf{S}
$$

This last equation highlights the relation between the single Basis Pursuit (when $L=1$ ) and the Multiple-Basis Pursuit algorithm presented here. Both algorithms lead to a shrinkage of the coefficient projection when considering orthonormal dictionary elements. With the inclusion of multiple signals, the shrinking factor becomes more robust to noise since it depends on the correlation of the atom $\phi_{i}$ to all signals.

This M-BCD algorithm can be considered as an extension to simultaneous signal approximations of the works of Sardy et al. 39] and Elad [12] which also considered block coordinate descent for single signal approximation. In addition to the works of Sardy et al. and Elad, many others authors have considered block-coordinate descent algorithm for related sparse approximation problems. For instance, block coordinate descent has also been used for solving the Lasso [19], and the elastic net [56].

\subsection{Landweber iterations}

For recovering vector valued data with joint sparsity constraints, Fornasier et al. 17] have proposed an extension of the Landweber iterative approach introduced by Daubechies et al. [9]. In their work, Fornasier et al. used an iterative shrinking algorithm (which has the flavor of a gradient projection approach) which is able to solve the general problem (4) with $p=1$ and $q=\{1,2, \infty\}$. We summarize here the details of their derivations for general $q$.

The problem to solve is the one given in Equation (4) with $p=1$ :

$$
\min _{\mathbf{C}} \frac{1}{2}\|\mathbf{S}-\mathbf{\Phi} \mathbf{C}\|_{F}^{2}+\lambda \sum_{i}\left\|c_{i, .}\right\|_{q}
$$


The iterative algorithm can be derived first by supposing that the objective function given above is strictly convex then by defining the following surrogate objective function :

$$
J_{s u r}(\mathbf{C}, \mathbf{A})=\frac{1}{2}\|\mathbf{S}-\mathbf{\Phi} \mathbf{C}\|_{F}^{2}+\lambda \sum_{i}\left\|c_{i, .}\right\|_{q}+\frac{1}{2}\|\mathbf{\Phi} \mathbf{C}-\mathbf{\Phi} \mathbf{A}\|_{F}^{2}-\frac{1}{2}\|\mathbf{C}-\mathbf{A}\|_{F}^{2}
$$

From this surrogate function, Fornasier et al. considers the solution of problem (4) as the limit of the sequence $\mathbf{C}^{(t)}$ :

$$
\mathbf{C}^{(t+1)}=\arg \min _{\mathbf{C}} J_{\text {sur }}\left(\mathbf{C}, \mathbf{C}^{(t)}\right)
$$

Note that according to the additional terms (the last two ones) in the surrogate function, $C^{(t+1)}$ is a coefficient matrix that minimizes the desired objective function plus terms that constraints $\mathbf{C}^{(t+1)}$ and the novel signal approximation $\mathbf{\Phi} \mathbf{C}^{(t+1)}$ to be "closed" respectively to $\mathbf{C}^{(t)}$ and the previous signal approximation $\mathbf{\Phi} \mathbf{C}^{(t)}$.

Now, the minimizer of $J_{\text {sur }}$ for fixed $\mathbf{A}$ can be obtained by expanding $J_{\text {sur }}$ as

$$
\begin{aligned}
J_{\text {sur }}(\mathbf{C}, \mathbf{A})= & \frac{1}{2}\left\|\left(\mathbf{A}+\boldsymbol{\Phi}^{t}(\mathbf{S}-\mathbf{\Phi} \mathbf{A})\right)-\mathbf{C}\right\|_{F}^{2}+\lambda \sum_{i}\left\|c_{i, .}\right\|_{q} \\
& -\frac{1}{2}\left\|\mathbf{A}+\boldsymbol{\Phi}^{t}(\mathbf{S}-\mathbf{\Phi} \mathbf{A})\right\|^{2}+\frac{1}{2}\|\mathbf{S}\|_{F}^{2}-\frac{1}{2}\|\mathbf{\Phi} \mathbf{A}\|_{F}^{2}+\frac{1}{2}\|\mathbf{A}\|_{F}^{2}
\end{aligned}
$$

and noticing that the second line of this equation does not depend on $\mathbf{C}$. Thus we have

$$
\arg \min _{\mathbf{C}} J_{\text {sur }}(\mathbf{C}, \mathbf{A})=U_{q}\left(\mathbf{A}+\boldsymbol{\Phi}^{t}(\mathbf{S}-\mathbf{\Phi} \mathbf{A})\right)
$$

with $U_{q}(\mathbf{X})$ being defined as

$$
U_{q}(\mathbf{X})=\arg \min _{\mathbf{C}} \frac{1}{2}\|\mathbf{X}-\mathbf{C}\|_{F}^{2}+\lambda \sum_{i}\left\|c_{i, .}\right\|_{q}
$$

Thus the iterative algorithm we get simply reads, at iteration $t$ as :

$$
\mathbf{C}^{(t+1)}=U_{q}\left(\mathbf{C}^{(t)}+\boldsymbol{\Phi}^{t}(\mathbf{S}-\boldsymbol{\Phi}) \mathbf{C}^{(t)}\right)
$$

The Landweber algorithm proposed by Fornasier et al. involves two steps : a first step which is similar to a gradient descent with fixed step and which does 


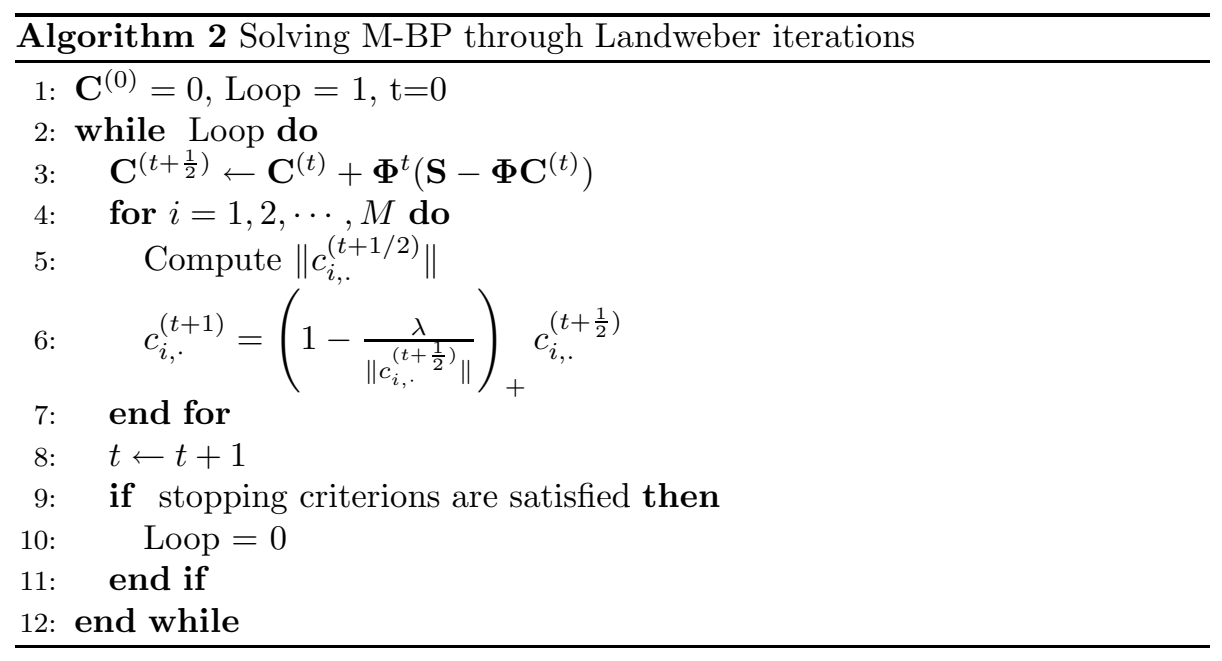

not take into account the sparsity constraint and a thresholding step through the operator $U_{q}(\cdot)$, which updates the solution according to the penalty $\sum_{i}\left\|c_{i, .}\right\|_{q}$. Fornasier et al. give detailed information of the $U_{q}(\cdot)$ closed form for $q=$ $\{1,2, \infty\}$. We can note that the problem (11) decouples with respect to the row of $\mathbf{C}$ and the thresholding operator $U_{q}(\cdot)$ acts independently on each row.

According to Fornasier et al. 17], for $q=2, U_{2}(\mathbf{C})$ writes for each row $c_{i,}$. as :

$$
\left[U_{2}(\mathbf{C})\right]_{i, \cdot}=\left(1-\frac{\lambda}{\left\|c_{i}\right\|}\right)_{+} c_{i,}
$$

and the full algorithm is summarized in Algorithm 2 We note that blockcoordinate descent and Landweber iterations yield to algorithms that are very similar. Two differences can be noted. The first one is that the shrinking operation for one case is based on $T_{i}$ while in the other case it directly considers $c_{i, .}$ At the moment, it is not clear what are the implications of these two different schemes in term of convergence rate and more investigations are needed to clarify this point. The other main difference between the two approaches is that, by optimizing at each loop, only the $c_{i}$.' $s$ that are not optimal yet, the BCD algorithm is more efficient than the one of Fornasier et al.

Note that Fornasier et al. have also shown that this iterative scheme converges towards the minimizer of problem (4) for $1 \geq q \geq \infty$. 


\subsection{Other works}

Besides the M-FOCUSS algorithm of Cotter et al., another seminal work which considered the SSA problem is the one of Malioutov et al. 29]. While dealing with a source localization problem, they had to consider a sparse approximation problem with joint sparsity constraints. From the block-sparse signal approximation problem

$$
\min _{\tilde{\mathbf{c}}} \frac{1}{2}\|\tilde{\mathbf{s}}-\tilde{\boldsymbol{\Phi}} \tilde{\mathbf{c}}\|_{2}+\lambda \sum_{i}^{M}\left\|\tilde{\mathbf{c}}_{g_{i}}\right\|_{2}
$$

they derived an equivalent problem which writes as :

$$
\begin{array}{ll}
\min _{p, q, \mathbf{r}, \tilde{\mathbf{c}}} & p+\lambda q \\
\text { st. } & \frac{1}{2}\|\tilde{\mathbf{s}}-\tilde{\mathbf{\Phi}} \tilde{\mathbf{c}}\|_{2}^{2} \leq p \\
& \sum_{i=1}^{M} r_{i} \leq q \\
& \left\|\mathbf{c}_{g_{i}}\right\| \leq r_{i} \quad \forall i=1, \cdots, M
\end{array}
$$

Note that this above problem has a linear objective function and linear and second-order cone constraints. Such a problem is known as a second-order cone programming problem and there exists interior point method for solving it 43]. While very attractive because of its simple formulation and the global convergence of the algorithm, this approach suffers from a high complexity which is of the order $\mathcal{O}\left(M^{3} L^{3}\right)$.

Very recently, two other algorithms have been proposed for addressing our jointly sparse approximation problem. The first one, derived by van den Berg et al. 50] is very similar to the one of Fornasier et al. although from a different perspective. Indeed, they develop a method based on spectral gradient projection for solving the Group lasso problem which is the one given in Equation (5) with $p=1$ and $q=2$. However, instead of the regularized version, they considered the following constrained version

$$
\begin{array}{ll}
\min _{\tilde{\mathbf{c}}} & \frac{1}{2}\|\tilde{\mathbf{s}}-\tilde{\boldsymbol{\Phi}} \tilde{\mathbf{c}}\|_{2} \\
\text { st. } & \sum_{i}^{M}\left\|\tilde{\mathbf{c}}_{g_{i}}\right\|_{2} \leq \tau
\end{array}
$$


that they iteratively solve owing to spectral gradient projection. Basically, their solution iterates write as

$$
\tilde{\mathbf{c}}^{(t+1)}=P\left(\tilde{\mathbf{c}}^{(t)}+\alpha \tilde{\boldsymbol{\Phi}}^{t}\left(\tilde{\mathbf{s}}-\tilde{\mathbf{\Phi}} \tilde{\mathbf{c}}^{(t)}\right)\right)
$$

where $\alpha$ is some step size to be optimized for instance by backtracking and $P(\cdot)$ is the projection operator defined as :

$$
P(\mathbf{z})=\left\{\arg \min _{\mathbf{x}}\|\mathbf{z}-\mathbf{x}\| \text { subject to } \sum_{i}\left\|\mathbf{x}_{g_{i}}\right\|_{2} \leq \tau\right\}
$$

van den Berg et al. then proposed an algorithm that computes this projection in linear time. Note how similar the iterates given in Equation (12) and (13) are. Actually, approaches of Fornasier et al. and van den Berg et al. are equivalent and the only point in which they differ is the way the projection are computed. This difference essentially comes from the problem formulation : Fornasier et al. use a penalized problem with known $\lambda$ while van den Berg consider the constrained optimization problem. Hence, the latter can not directly use the analytic solution of the projection $P(\cdot)$ but have to compute the appropriate value of $\lambda$ given their value of $\tau$. However, the algorithm they derive for this projection is efficient. Note that if one has some prior knowledge on the value of $\tau$, then this algorithm would be preferable than the one of Fornasier et al.

The other recent work that is noteworthy is the one of Obozinski et al. 33]. They address the problem of SSA within the context of multi-task classification problem. Indeed, they want to recover a common set of covariates that are simultaneously relevant for all classification tasks. For achieving this aim, they propose to solve problem (4) with $p=1, q=2$ and a differentiable loss function such as the logistic loss or the square loss function. One of their most prominent contribution is to have proposed a path-following algorithm that is able to compute the regularization path of the solution with respects to $\lambda$. Their algorithm uses a continuation method based on prediction-correction steps. The prediction step is built upon the optimality conditions given in equation (7) while the correction step needs an algorithm which solves problem (4) but only for a small 
subset of covariates. The main advantage of such an algorithm is its efficiency for selecting an appropriate value of $\lambda$ in a model selection context.

\section{Generic algorithms for large classes of $p$ and $q$}

In this section, we present several algorithms that are able to solve the simultaneous sparse approximation problem for a large classes of $p$ and $q$. In the first part of the section, we review the M-FOCUSS algorithm of Cotter et al. 8] that addresses the case where $0<p \leq 1$ and $q=2$. In a second part, we make clear how the penalization term $J_{p, q}(\mathbf{C})$ with $p=1$ and $1 \leq q \leq 2$, is related to automatic relevance determination. From this novel insight, we then propose an iterative reweighted least-square algorithm that solves this general case. The last part of the section is devoted to the extension of reweighted $\ell_{1}$ algorithms 57, 4] to the SSA problem. We describe how algorithms tailored for solving the SSA problem with $p=1$ and any $q$ can be re-used for solving a problem with the same $q$ but $p<1$.

\subsection{M-FOCUSS algorithm}

We first detail how the M-FOCUSS algorithm proposed by Cotter et al. [8] can be derived, then we discuss some of its properties and relation with other works.

\subsubsection{Deriving the algorithm}

M-FOCUSS is, as far as we know, the first algorithm that has been introduced for solving simultaneous sparse approximation problem. M-FOCUSS addresses the general case where $q=2$ and $p \leq 1$. This algorithm can be understood as a fixed-point algorithm which can be derived through an appropriate factorization of problem (4) objective function gradient.

Indeed, since the partial derivative of $J_{p, 2}(\mathbf{C})$ with respects to an entry $c_{m, n}$ is :

$$
\begin{aligned}
\frac{\partial J_{p, 2}(\mathbf{C})}{\partial c_{m, n}} & =\frac{\partial}{\partial c_{m, n}} \sum_{i}\left(\sum_{j} c_{i, j}^{2}\right)^{p / 2} \\
& =p\left\|c_{m, \cdot}\right\|_{2}^{p-2} c_{m, n}
\end{aligned}
$$


the gradient of the objective function writes:

$$
-\boldsymbol{\Phi}^{t}(\mathbf{S}-\mathbf{\Phi C})+\lambda \mathbf{P C}
$$

where $\mathbf{P}=\operatorname{diag}\left(p\left\|c_{i},\right\|_{2}^{p-2}\right)$. Then, we define the weighting matrix $\mathbf{W}$ as $\mathbf{W}=$ $\operatorname{diag}\left(p^{-1 / 2}\left\|c_{i, \cdot}\right\|_{2}^{1-p / 2}\right)$. After having replaced $\mathbf{W}^{-2}=\mathbf{P}$ in the above gradient, and after simple algebras, we have the following necessary optimality condition

$$
\left((\mathbf{\Phi} \mathbf{W})^{t}(\mathbf{\Phi} \mathbf{W})+\lambda \mathbf{I}\right) \mathbf{W}^{-1} \mathbf{C}=(\mathbf{\Phi} \mathbf{W})^{t} \mathbf{S} .
$$

Hence, the optimum solution writes as

$$
\mathbf{C}^{\star}=\mathbf{W}\left((\boldsymbol{\Phi} \mathbf{W})^{t}(\mathbf{\Phi} \mathbf{W})+\lambda \mathbf{I}\right)^{-1}(\mathbf{\Phi} \mathbf{W})^{t} \mathbf{S} .
$$

Note that since $\mathbf{W}$ also depends on $\mathbf{C}$, the above closed-form expression of the optimal matrix $\mathbf{C}$ can be interpreted as a fixed-point algorithm. From this insight on $\mathbf{C}$, Cotter et al. suggest the following iterates for solving the simultaneous sparse approximation problem :

$$
\mathbf{C}^{(t+1)}=\mathbf{W}^{(t)}\left(\left(\boldsymbol{\Phi} \mathbf{W}^{(t)}\right)^{t}\left(\mathbf{\Phi} \mathbf{W}^{(t)}\right)+\lambda \mathbf{I}\right)^{-1}\left(\boldsymbol{\Phi} \mathbf{W}^{(t)}\right)^{t} \mathbf{S}
$$

where $\mathbf{W}^{(t)}=\operatorname{diag}\left(p^{-1 / 2}\left\|c_{i, \cdot}^{(t)}\right\|_{2}^{1-p / 2}\right)$. Now, since we have :

$\left(\left(\mathbf{\Phi} \mathbf{W}^{(t)}\right)^{t}\left(\mathbf{\Phi} \mathbf{W}^{(t)}\right)+\lambda \mathbf{I}\right)^{-1}\left(\mathbf{\Phi} \mathbf{W}^{(t)}\right)^{t}=\left(\mathbf{\Phi} \mathbf{W}^{(t)}\right)^{t}\left(\left(\mathbf{\Phi} \mathbf{W}^{(t)}\right)\left(\mathbf{\Phi} \mathbf{W}^{(t)}\right)^{t}+\lambda \mathbf{I}\right)^{-1}$

Cotter et al. actually consider the following iterative scheme

$$
\mathbf{C}^{(t+1)}=\mathbf{W}^{(t)}\left(\boldsymbol{\Phi} \mathbf{W}^{(t)}\right)^{t}\left(\left(\boldsymbol{\Phi} \mathbf{W}^{(t)}\right)\left(\boldsymbol{\Phi} \mathbf{W}^{(t)}\right)^{t}+\lambda \mathbf{I}\right)^{-1} \mathbf{S}
$$

Resulting algorithm is detailed in Algorithm 3

\subsubsection{Discussing M-FOCUSS}

Rao et al 37] has provided an interesting interpretation on the FOCUSS algorithm for a single signal approximation which can be readily extend to MFOCUSS. Indeed, M-FOCUSS can be viewed as an iterative reweighted leastsquare algorithm. Indeed, From the updating equation of $\mathbf{C}^{(t)}$ (see Equation (16)) , we can note that $\mathbf{C}^{(t)}=\mathbf{W}^{(t)} \mathbf{Z}^{(t)}$ where $\mathbf{Z}$ is the minimizer of

$$
\frac{1}{2}\left\|\mathbf{S}-\mathbf{\Phi} \mathbf{W}^{(t)} \mathbf{Z}\right\|_{F}^{2}+\frac{\lambda}{2}\|\mathbf{Z}\|_{F}^{2}
$$




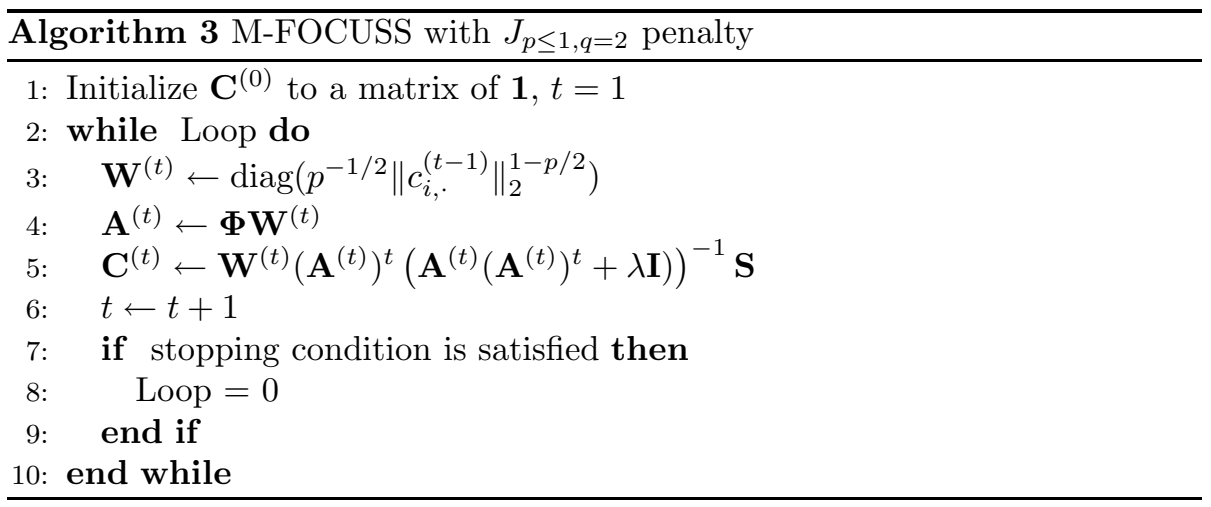

and thus $\mathbf{C}^{(t)}$ can be understood as the minimizer of

$$
\frac{1}{2}\|\mathbf{S}-\mathbf{\Phi} \mathbf{C}\|_{F}^{2}+\frac{\lambda}{2}\left\|\left(\mathbf{W}^{(t)}\right)^{-1} \mathbf{C}\right\|_{F}^{2} .
$$

This above equation makes clear the relation between sparse approximation problem and iterative reweighted least-square. Such an connection has already been highlighted in other context. Indeed, while sparsity is usually induced by using $\ell_{1}$ norm penalty, it has been proved that solving the problem in which the $\ell_{1}$ norm has been replaced by an adaptive $\ell_{2}$ norm leads to equivalent solutions 10, 38, 22].

Despite this nice insight, M-FOCUSS presents an important issue. Indeed, the updates proposed by Cotter et al. are not guaranteed to converge to a local minimum of the problem (if the problem is not convex $p<1$ ) or to the global minimum of the convex problem $(p=1)$. Their algorithm presents several fixed-points since when a row of $\mathbf{C}$ is equal to 0 , it stays at 0 at the next iteration. Although such a point may be harmless if the algorithm is initialized with a "good" starting point, it is nonetheless an undesirable point when solving a convex problem. At the contrary, the M-BCD and the Landweber iteration based algorithms do not suffer from the presence of such fixed points. However, in the M-FOCUSS algorithm, this pathological behavior can be handled by introducing a smoothing term $\varepsilon$ in the weight so that the updated weight becomes

$$
\left.\mathbf{W}^{(t)}=\operatorname{diag}\left(p^{-1 / 2}\left(\left\|c_{i, \cdot}^{(t)}\right\|+\epsilon\right)\right)^{1-p / 2}\right)
$$


where $\varepsilon>0$. The use of $\varepsilon$ avoids a given weight to be at zero and consequently it avoids the related $c_{i}$. to stay permanently at zero. If we furthermore note that M-FOCUSS is not more than an iterative reweighted least-square, then according to the very recent works of Daubechies et al. 10] and Chartrand et al. 5], it seems justified to iterate the M-FOCUSS algorithm using decreasing value of $\varepsilon$. In our numerical experimental, we will consider the M-FOCUSS algorithm with fixed and decreasing value of $\varepsilon$.

\subsection{Automatic relevance determination approach}

In this section, we focus on the relaxed optimization problem given in (4) with the general penalization $J_{p, q}(\mathbf{C})$. Our objective here is to clarify the connection between such a form of penalization and the automatic relevance determination of C's rows, which has been the keystone of the Bayesian approach of Wipf et al [54]. We will show that for some values of $p$ and $q$, the mixed-norm $J_{p, q}(\mathbf{C})$ has an equivalent variational formulation. Then, by using this novel formulation in problem (4), instead of $J_{p, q}(\mathbf{C})$, we exhibit the relation between

our sparse approximation problem and ARD. We then propose an iterative reweighted least-square approach for solving this resulting ARD problem.

\subsubsection{Exhibiting the relation with $A R D$}

For this purpose, we first consider the following formulation of the simultaneous sparse approximation problem:

$$
\min _{\mathbf{C}} \frac{1}{2}\|\mathbf{S}-\mathbf{\Phi} \mathbf{C}\|_{F}^{2}+\lambda^{\prime}\left(J_{p, q}(\mathbf{C})\right)^{\frac{2}{p}} .
$$

In the convex case (for $p \geq 1$ and $q \geq 1$ ), since the power function is strictly monotonically increasing, problems (4) and (18) are equivalent, in the sense that for a given value $\lambda^{\prime}$, there exists a $\lambda$ so that solutions of the two problems are equivalent. When $J_{p, q}$ is not convex, this equivalence does not strictly apply. However, due to the nature of the problem, the problem formulation (18) is more convenient for exhibiting the relation with ARD.

Let us introduce the key lemma that allows us to derive the ARD-based formulation of the problem. This lemma gives a variational form of the $\ell_{p, q}$ norm of a sequence $\left\{a_{t, k}\right\}$. 
Lemma 1. if $s>0$ and $\left\{a_{t, k}: k \in \mathbb{N}_{n}, t \in \mathbb{N}_{T}\right\} \in \mathbb{R}$ such that at least one $a_{t, k}>0$, then

$$
\min _{\mathbf{d}}\left\{\sum_{t, k} \frac{\left|a_{t, k}\right|^{2}}{d_{t, k}}: d_{t, k} \geq 0, \sum_{k}\left(\sum_{t} d_{t, k}^{1 / s}\right)^{\frac{s}{r+s}} \leq 1\right\}=\left(\sum_{k}\left(\sum_{t}\left|a_{t, k}\right|^{q}\right)^{\frac{p}{q}}\right)^{\frac{2}{p}}
$$

where $q=\frac{2}{s+1}$ and $p=\frac{2}{s+r+1}$. Furthermore, at optimality, we have:

$$
d_{t, k}^{\star}=\frac{\left|a_{t, k}\right|^{\frac{2 s}{s+1}}\left(\sum_{u}\left|a_{u, k}\right|^{\frac{2}{s+1}}\right)^{\frac{r}{s+r+1}}}{\left(\sum_{v}\left(\sum_{u}\left|a_{u, v}\right|^{\frac{2}{s+1}}\right)^{\frac{s+1}{s+r+1}}\right)^{r+s}}
$$

Proof : the proof has been post-poned to the appendix.

According to this lemma, the $\ell_{p, q}$ norm of a sequence can be computed through a minimization problem. Hence, applying this lemma to $\left(J_{p, q}(\mathbf{C})\right)^{\frac{2}{p}}$ by defining $a_{t, k}=c_{t, k}$, we get a variational form of the penalization term. We can also note that the mixed-norm on the matrix coefficients has been transformed to a mixed-norm on weight matrix $\mathbf{d}$. Plugging the above variational formulation of the penalization term in problem (18) yields to the following equivalent problem :

$$
\begin{array}{ll}
\min _{\mathbf{C}, \mathbf{d}} & \frac{1}{2}\|\mathbf{S}-\mathbf{\Phi} \mathbf{C}\|_{F}^{2}+\lambda \sum_{t, k} \frac{c_{t, k}^{2}}{d_{t, k}} \\
\text { s.t. } & \sum_{k}\left(\sum_{t} d_{t, k}^{1 / s}\right)^{\frac{s}{r+s}} \leq 1 \\
& d_{t, k} \geq 0 \quad \forall t, k
\end{array}
$$

This problem is the one which makes clear the automatic relevance determination interpretation of the original formulation (4). Indeed, we have transformed problem (4) into a problem with a smooth objective function at the expense of some additional variables $d_{t, k}$. These parameters $d_{t, k}$ actually aim at determining the relevance of each element of $\mathbf{C}$. Indeed, in the objective function, each squared-value $c_{t, k}$ is now inversely weighted by a coefficient $d_{t, k}$. By taking the convention that $\frac{x}{0}=\infty$ if $x \neq 0$ and 0 otherwise, the objective value of the optimization problem becomes finite only if $d_{t, k}=0$ for $c_{t, k}^{2}=0$. Then the smaller $d_{t, k}$ is, the smaller the $c_{t, k}$ norm should be. Furthermore, optimization 
problem (21) also involves some constraints on $\left\{d_{t, k}\right\}$. These constraints impose the matrix $\mathbf{d}$ to have positive elements and to be so that its $\ell_{\frac{1}{r+s}, \frac{1}{s}}$ mixed-norm is smaller than 1 . Note that this mixed-norm on $\mathbf{d}$ plays an important role since it induces the row-norm sparsity on $\mathbf{C}$. According to the relation between $p, r$ and $s$, for $p<1$, we also have $r+s>1$, making the $\ell_{\frac{1}{r+s}, \frac{1}{s}}$ non-differentiable with respect to the first norm. Such singularities favor row-norm sparsity of the matrix $\mathbf{d}$ at optimality, inducing row-norm sparsity of $\mathbf{C}$. As we have noted above, when a row-norm of $\mathbf{d}$ is equal to 0 , the corresponding row-norm of $\mathbf{C}$ should also be equal to 0 which means that the corresponding element of the dictionary is "irrelevant" for the approximation of all signals. Problem (21) proposes an equivalent formulation of problem (44) for which the row-diversity measure has been transformed in another penalty function owing to an ARD formulation. The trade-off between convexity of the problem and the sparsity of the solution has been transferred from $p$ and $q$ to $r$ and $s$.

From a Bayesian perspective, we can interpret the mixed-norm on $\mathbf{d}$ as the diagonal term of the covariance matrix of a Gaussian prior over the row-norm on $\mathbf{C}$ distribution. This is typically the classical Bayesian Automatic Relevance Determination approach as proposed for instance by Tipping [46]. This novel insight on the ARD interpretation of $J_{p, q}(\mathbf{C})$ clarifies the connection between the M-FOCUSS algorithm of Cotter et al. [8] and the Multiple Sparse Bayesian Learning (M-SBL) algorithm of Wipf et al. 54] for any value of $p<1$. In their previous works, Wipf et al. have proved that these two algorithms were related when $p \approx 0$. Here, we refine their result by enlarging the connection to other values of $p$ by showing that both algorithms actually solve a problem with automatic relevance determination on the row-norm of $\mathbf{C}$.

\subsection{Solving the ARD formulation for $p=1$ and $1 \leq q \leq 2$}

Herein, we propose a simple iterative algorithm for solving problem (21)

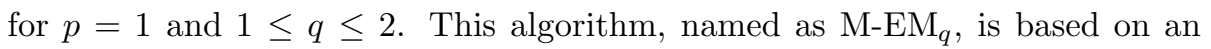
iterative-reweighted least squares where the weights are updated according to equation (20). Thus, it can be seen as an extension of the M-FOCUSS algorithm 


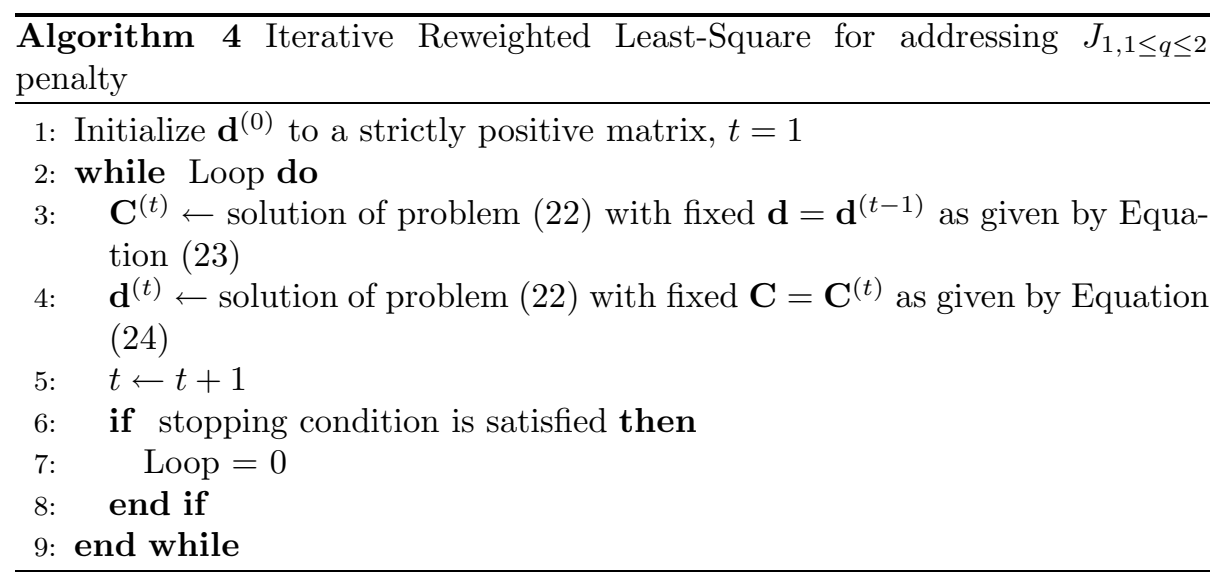

of Cotter et al. for $q \leq 2$. Note that we have restricted ourselves to $p=1$ since we will show in the next section that the case $p<1$ can be handled using another reweighted scheme.

Since $p=1$, thus $s+r=1$, the problem we are considering is :

$$
\begin{array}{ll}
\min _{\mathbf{C}, \mathbf{d}} & \sum_{k} \frac{1}{2}\left(\left\|\mathbf{s}_{k}-\mathbf{\Phi} c_{\cdot, k}\right\|_{2}^{2}+\lambda \sum_{t} \frac{c_{t, k}^{2}}{d_{t, k}}\right)=J_{o b j}(\mathbf{C}, \mathbf{d}) \\
\text { s.t. } & \sum_{k}\left(\sum_{t} d_{t, k}^{1 / s}\right)^{s} \leq 1 \\
& d_{t, k} \geq 0
\end{array}
$$

Since, we consider that $1 \leq q \leq 2$ hence $0 \leq s \leq 13$, this optimization problem is convex with a smooth objective function. We propose to address this problem through a block-coordinate algorithm which alternatively solves the problem with respects to $\mathbf{C}$ with the weight $\mathbf{d}$ being fixed, and keeping $\mathbf{C}$ fixed and computing the optimal weight $\mathbf{d}$. The resulting algorithm is detailed in Algorithm 4

Owing to the problem structure, step 4 and 5 of this algorithm has a simple closed form. Indeed, for fixed $\mathbf{d}$, each vector $c_{\cdot, k}^{(t)}$ at iteration $t$ is given by :

$$
c_{\cdot, k}^{(t)}=\left(\boldsymbol{\Phi}^{t} \boldsymbol{\Phi}+2 \lambda \mathbf{D}_{k}^{(t-1)}\right)^{-1} \boldsymbol{\Phi}^{t} \mathbf{s}_{k}
$$

where $\mathbf{D}_{k}^{(t-1)}$ is a diagonal matrix of entries $1 / d_{\cdot, k}^{(t-1)}$. In a similar way, for fixed $\mathbf{C}$, step 5 boils down in solving problem (19). Hence, by defining $a_{t, k}=c_{t, k}^{(t)}$, we

\footnotetext{
${ }^{3}$ for $s=0$, we have explicitly used the sup norm of vector $d_{,, k}$ in the constraints.
} 
also have a closed-form for $\mathbf{d}^{(t)}$ as

$$
d_{t, k}^{(t)}=\frac{\left|a_{t, k}\right|^{\frac{2 s}{s+1}}\left(\sum_{u}\left|a_{u, k}\right|^{\frac{2}{s+1}}\right)^{\frac{1-s}{2}}}{\sum_{v}\left(\sum_{u}\left|a_{u, v}\right|^{\frac{2}{s+1}}\right)^{\frac{s+1}{2}}}
$$

Note that similarly to the M-FOCUSS algorithm, this algorithm can also be seen as an iterative reweighted least-square approach or as an ExpectationMinimization algorithm, where the weights are defined in equation (24). Furthermore, it can be shown that if the weights $\mathbf{d}$ are initialized to non-zero values then at each loop involving step 4 and 5, the objective value of problem (22) decreases. Hence, since the problem is convex, our algorithm should converge towards the global minimum of the problem.

Theorem 2. If the objective value of problem (22) is strictly convex (for instance when $\mathbf{\Phi}$ is full-rank), and if for the $t$-th loop, after the step 5, we have $\mathbf{d}^{(t)} \neq \mathbf{d}^{(t-1)}$, then the objective value has decreased, i.e :

$$
J_{o b j}\left(\mathbf{C}^{(t+1)}, \mathbf{d}^{(t)}\right)<J_{o b j}\left(\mathbf{C}^{(t)}, \mathbf{d}^{(t)}\right)<J_{o b j}\left(\mathbf{C}^{(t)}, \mathbf{d}^{(t-1)}\right) .
$$

Proof: The right inequality $J_{o b j}\left(\mathbf{C}^{(t)}, \mathbf{d}^{(t)}\right)<J_{o b j}\left(\mathbf{C}^{(t)}, \mathbf{d}^{(t-1)}\right)$ comes from $\mathbf{d}^{(t)}$ being the optimal value of the optimization problem resulting from step 5 of algorithm (4). The strict inequality yields from the hypothesis that $\mathbf{d}^{(t)} \neq \mathbf{d}^{(t-1)}$ and from the strict convexity of the objective function. A similar reasoning allows us to derive the left inequality. Indeed, since $\mathbf{C}^{(t)}$ is not optimal with respects to $\mathbf{d}^{(t)}$ for the problem given by step (4), invoking the strict convexity of the associated objective function and optimality of $\mathbf{C}^{(t+1)}$ concludes the proof.

As stated by the above theorem, the decrease in objective value is actually guaranteed unless, the algorithm get stuck in some fixed points (e.g all the elements of $\mathbf{d}$ being zero expect for one entry $\left.\left\{t_{1}, k_{1}\right\}\right)$. In practice, we have experienced, by comparing for $q=2$ with the M-BCD algorithm, that if $\mathbf{d}$ is initialized to non-zero entries, algorithm (4) converges to the global minimum of problem (22). Numerical experiments will illustrate this point. 


\subsection{Iterative reweighted $\ell_{1}-\ell_{q}$ algorithms for $p<1$}

This section introduces an iterative reweighted M-Basis Pursuit (IrM-BP) algorithm and proposes a way for setting the weight. Through this approach, we are able to provide an iterative algorithm which solves problem (4) with $p<1$ and $1 \leq q \leq 2$.

\subsubsection{Iterative reweighted algorithm}

Recently, several works have advocated that sparse approximations can be recovered through iterative algorithms based on a reweighted $\ell_{1}$ minimization 57, 4, 5, 52, 18]. Typically, for a single signal case, the idea consists in iteratively solving the following problem

$$
\min _{\mathbf{c}} \frac{1}{2}\|\mathbf{s}-\mathbf{\Phi} \mathbf{c}\|_{2}^{2}+\lambda \sum_{i} z_{i}\left|c_{i}\right|
$$

where $z_{i}$ are some positive weights, and then to update the positive weights $z_{i}$ according to the solution $\mathbf{c}^{\star}$ of the above problem. Besides providing empirical evidences that reweighted $\ell_{1}$ minimization yields to sparser solutions than a simple $\ell_{1}$ minimization, the above cited works theoretically support such a claim. These results for the single signal approximation case suggest that in the simultaneous sparse approximation problem, reweighted M-Basis Pursuit would also lead to sparser solutions than the classical M-Basis Pursuit.

The iterative reweighted M-Basis Pursuit algorithm is defined as follows. We iteratively construct a sequence $\mathbf{C}^{(t)}$ defined as

$$
\mathbf{C}^{(t)}=\arg \min _{\mathbf{C}} \frac{1}{2}\|\mathbf{S}-\mathbf{\Phi} \mathbf{C}\|_{F}^{2}+\lambda \sum_{i} z_{i}^{(t)}\left\|c_{i, \cdot}\right\|_{q}
$$

where the positive weight vector $\mathbf{z}^{(t)}$ depends on the previous iterate $\mathbf{C}^{(t-1)}$. For $t=1$, we typically define $\mathbf{z}^{(1)}=1$ and for $t>1$, we will consider the following weighting scheme

$$
z_{i}^{(t)}=\frac{1}{\left(\left\|c_{i,}^{(t-1)}\right\|_{q}+\varepsilon\right)^{r}} \quad \forall i
$$

where $\left\{c_{i, \cdot}^{(t-1)}\right\}$ is the i-th row of $\mathbf{C}^{(t-1)}, r$ a user-defined positive constant and $\varepsilon$ a small regularization term that prevents from having an infinite regularization 


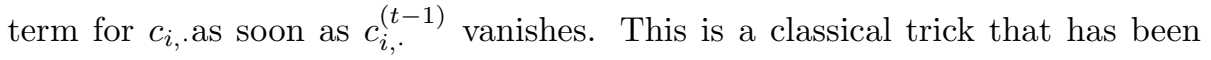
used for instance by Candès et al. [4] or Chartrand et al. 38]. Note that for any positive weight vector $\mathbf{z}$, problem (25) is a convex problem that does not present local minima. Furthermore, for $1 \leq q \leq 2$, it can be solved by our

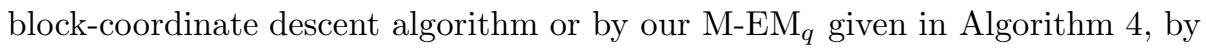
simply replacing $\lambda$ with $\lambda_{i}=\lambda \cdot z_{i}$. This reweighting scheme is similar to the adaptive lasso algorithm of Zou et al. 57] but uses a larger number of iterations and addresses the simultaneous approximation problem.

\subsubsection{Connections with Majorization-Minimization algorithm}

This IrM-BP algorithm can be interpreted as an algorithm for solving an approximation of problem (4) when $0<p<1$ and $1 \leq q \leq 2$. Indeed, similarly to the reweighted $\ell_{1}$ scheme of Candès et al. 4] or the one-step reweighted lasso of Zou et al. 58], this algorithm falls into the class of majorize-minimize (MM) algorithms 24]. MM algorithms consists in replacing a difficult optimization problem with a easier one, for instance by linearizing the objective function, by solving the resulting optimization problem and by iterating such a procedure.

The connection between MM algorithms and our reweighted scheme can be made through linearization. Let us first define $J_{p, q, \varepsilon}(\mathbf{C})$ as an approximation of the penalty term $J_{p, q}(\mathbf{C})$ :

$$
J_{p, q, \varepsilon}(\mathbf{C})=\sum_{i} g\left(\left\|c_{i, \cdot}\right\|_{q}+\varepsilon\right)
$$

where $g(\cdot)=|\cdot|^{p}$. Since $g(\cdot)$ is concave for $0<p<1$, a linear approximation of $J_{p, q, \varepsilon}(\mathbf{C})$ around $\mathbf{C}^{(t-1)}$ yields to the following majorizing inequality

$$
J_{p, q, \varepsilon}(\mathbf{C}) \leq J_{p, q, \varepsilon}\left(\mathbf{C}^{(t-1)}\right)+\sum_{i} \frac{p}{\left(\left\|c_{i, \cdot}^{(t-1)}\right\|_{q}+\varepsilon\right)^{1-p}}\left(\left\|c_{i, \cdot}\right\|_{q}-\left\|c_{i, \cdot}^{(t-1)}\right\|_{q}\right)
$$

then for the minimization step, replacing in problem (4) $J_{p, q}$ with the right part of the inequality and dropping constant terms lead to our optimization problem (25) with appropriately chosen $z_{i}$ and $r$. Note that for the weights given in equation (26), $r=1$ corresponds to the linearization of a log penalty 


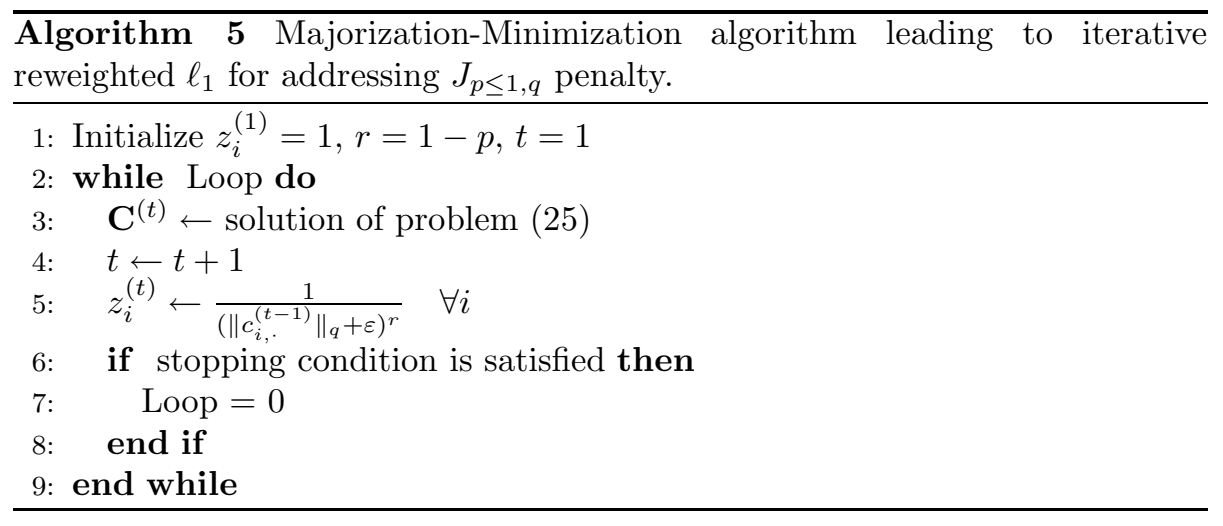

$\sum_{i} \log \left(\left\|c_{i,}\right\|+\varepsilon\right)$ whereas setting $r=1-p$ corresponds to a $\ell_{p}$ penalty $(0<$ $p<1)$.

MM algorithms have already been considered in optimization problems with sparsity-inducing penalties. For instance, an MM approach has been used by Figueiredo et al. [16] for solving a least-square problem with a $\ell_{p}$ sparsityinducing penalty, whereas Candès et al. 44 have addressed the problem for exact sparse signal recovery. In a context of simultaneous approximation, Simila 40, 41] has also considered MM algorithms while approximating the nonconvex penalty with a quadratic term. In the light of all these previous works, what we have exposed here is merely an extension of iterative reweighted $\ell_{1}$ algorithm to simultaneous sparse approximation problem. Through this iterative scheme, we can solve problem (4) with $p<1$ and any $q$ provided that we have an algorithm that is able to solve problem (4) with $p=1$ and the desired $q$.

Analyzing the convergence of the sequence $\mathbf{C}^{(t)}$ towards the global minimizer of problem (4) is a challenging issue. Indeed, several points make a formal proof of convergence difficult. At first, in order to avoid a row $c_{i, \text {. }}^{(t)}$ to be permanently at zero, we have introduced a smoothing term $\varepsilon$, thus we are only solving a $\varepsilon$ approximation of problem (4). Furthermore, the penalty we use is non-convex, thus using a monotonic algorithm like a MM approach which decreases the objective value at each iteration, can not guarantee convergence to the global 
minimum of our $\varepsilon$-approximate problem. Hence, due to these two major obstacles, we have left this convergence proof for future works. Note however that few works have addressed the convergence issue of reweighted $\ell_{1}$ or $\ell_{2}$ algorithms for single sparse signal recovery. Notably, we can mention the recent work of Daubechies et al. 10] which provide a convergence proof of iterative reweighted least square for exact sparse recovery. In the same flavor, Foucart et al. 18] have proposed a tentative of rigorous convergence proof for reweighted $\ell_{1}$ sparse signal recovery. Although, we do not have any rigorous proof of convergence, in practice, we will show that the reweighted algorithm provides good sparse approximations.

As already noted by several authors $[4,38,10], \varepsilon$ plays a major role in the quality of the solution. In the experimental results presented below, we have investigated two methods for setting $\varepsilon$ : the first one is to set it to a fixed value $\varepsilon=0.001$, the other one, denoted as an annealing approach, consists in gradually decreasing $\varepsilon$ after having solved problem (25).

\subsubsection{Connection with group bridge Lasso}

Recently, in a context of variable selection through Group Lasso, Huang et al. 23] have proposed an algorithm for solving problem (51) with $p<1$ and $q=1$ :

$$
\min _{\tilde{\mathbf{c}}} \frac{1}{2}\|\tilde{\mathbf{s}}-\tilde{\mathbf{\Phi}} \tilde{\mathbf{c}}\|^{2}+\lambda \sum_{i}\left\|\tilde{\mathbf{c}}_{g_{i}}\right\|_{1}^{p} .
$$

Instead of directly addressing this optimization problem, they have shown that the above problem is equivalent to the minimization problem :

$$
\begin{array}{ll}
\min _{\tilde{\mathbf{c}}, \mathbf{z}} \quad \frac{1}{2}\|\tilde{\mathbf{s}}-\tilde{\boldsymbol{\Phi}} \tilde{\mathbf{c}}\|^{2}+\sum_{i} \frac{\left\|\tilde{\mathbf{c}}_{g_{i}}\right\|_{1}}{z_{i}^{1 / p-1}}+\tau \sum_{i} z_{i} \\
\text { st. } & z_{i} \geq 0 \quad \forall i .
\end{array}
$$

where $\tau$ is a regularization parameter. Here equivalence is understood as follows : $\tilde{\mathbf{c}}^{\star}$ minimizes equation (27) if and only if the pair $\left(\tilde{\mathbf{c}}^{\star}, \mathbf{z}^{\star}\right)$ minimizes Equation (27) with an appropriate value of $\tau$. This value of $\tau$ will be made clear in the sequel.

The connection between iterative reweighted $\ell_{1}$ approach and the algorithm proposed by Huang et al. comes from the way problem (28) has been solved. 


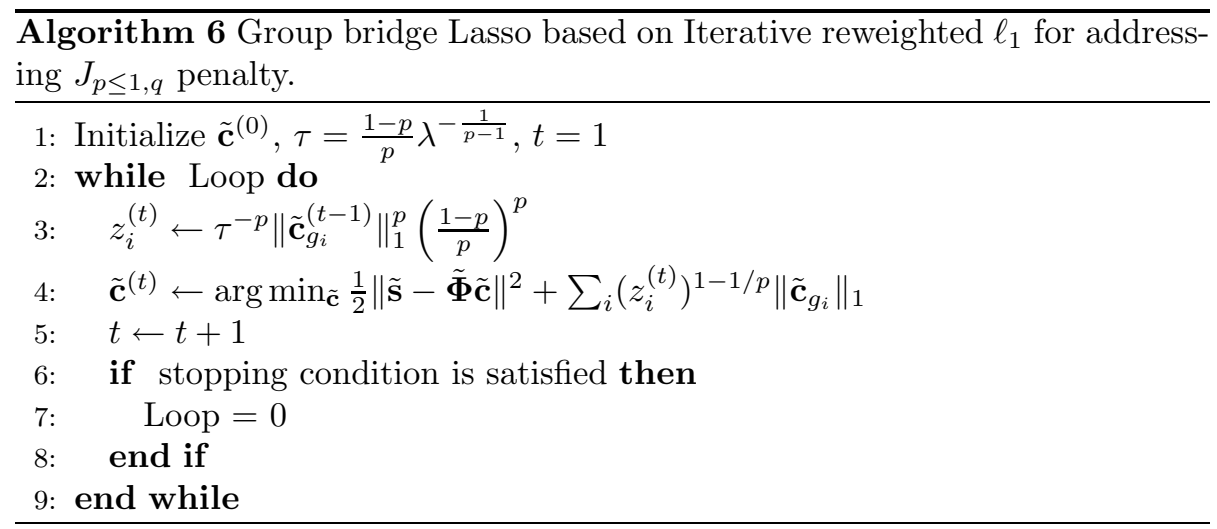

Indeed, Huang et al. have considered a block-coordinate approach which consists in minimizing the problem with respects to $\mathbf{z}$ and then, after having plugged the optimal $\mathbf{z}$ in the equation (28) in minimizing the problem with respects to $\tilde{\mathbf{c}}$. For the first step, the optimal $\mathbf{z}$ is derived by minimizing the convex problem

$$
\begin{array}{ll}
\min _{\mathbf{z}} \quad \sum_{i} \frac{\left\|\tilde{\mathbf{c}}_{g_{i}}\right\|_{1}}{z_{i}^{1 / p-1}}+\tau \sum_{i} z_{i} \\
\text { st. } & z_{i} \geq 0 \quad \forall i .
\end{array}
$$

Using Lagrangian theory, simple algebras yield to a closed-form solution of the optimal $\mathbf{z}$ :

$$
z_{i}^{\star}=\tau^{-p}\left\|\tilde{\mathbf{c}}_{g_{i}}\right\|_{1}^{p}\left(\frac{1-p}{p}\right)^{p}
$$

Plugging these $z_{i}$ 's in Equation (28) and using $\tau$ such that $\lambda=\left(\frac{1-p}{p \tau}\right)^{p-1}$ proves the equivalence of the problems (27) and (28). The relation with iterative reweighted $\ell_{1}$ is made clear in the second step of the block-coordinate descent. Indeed, in problem (28), since we only optimize with respects to $\tilde{\mathbf{c}}$ with fixed $z_{i}$, this step is equivalent to

$$
\min _{\tilde{\mathbf{c}}} \quad \frac{1}{2}\|\tilde{\mathbf{s}}-\tilde{\boldsymbol{\Phi}} \tilde{\mathbf{c}}\|^{2}+\sum_{i} \frac{\left\|\tilde{\mathbf{c}}_{g_{i}}\right\|_{1}}{z_{i}^{1 / p-1}}
$$

which is clearly a weighted $\ell_{1}$ problem. The full iterative reweighted algorithm is detailed in Algorithm (6). Note that although the genuine work on Group bridge Lasso only addresses the case $q=1$ (because of theoretical developments considered in the paper), the algorithm proposed by Huang et al. can be applied 
to any choice of $q$ provided that one is able to solve the related $\ell_{1}-\ell_{q}$ problem (for instance using the algorithm described in Section 3 ).

\section{Specific case algorithms}

In this section, we survey some algorithms that provide solutions of the SSA problem (4) when $p=0$. These algorithms are different in nature and provide different qualities of approximation. The first two algorithms we detail are greedy algorithms which have the flavor of Matching Pursuit [30. The third one is based on a sparse bayesian learning and is related to iterative reweighted $\ell_{1}$ algorithm.

\section{1. $S-O M P$}

Simultaneous Orthogonal Matching Pursuit (S-OMP) 48 is an extension of the Orthogonal Matching Pursuit [34, 47] algorithm to the multiple approximation problem.

$\mathrm{S}-\mathrm{OMP}$ is a greedy algorithm which is based on the idea of selecting, at each iteration, an element of the dictionary and on building all signal approximations as the projection of the signal matrix $\mathbf{S}$ on the span of these selected dictionary elements. Since, according to problem (2), one looks for a for dictionary elements that can simultaneously provide good approximation of all signals, Tropp et al. have suggested to select the dictionary element that maximizes the sum of absolute correlation between the basis element and the signal residuals. This greedy selection criterion is formalized as follows :

$$
\max _{i} \sum_{k=1}^{L}\left|\left\langle\mathbf{s}_{k}-P^{(t)}\left(\mathbf{s}_{k}\right), \boldsymbol{\Phi}_{i}\right\rangle\right|
$$

where $P^{(t)}(\cdot)$ is the projection operator on the span of the $t$ selected dictionary elements. While conceptually very simple, this algorithm which details are given in Algorithm (7), provide theoretical guarantees about the quality of its approximation. For instance, Tropp et al. [48] have shown that if the algorithm is stopped when $T$ dictionary elements have been selected then, under some mild conditions on the matrix $\boldsymbol{\Phi}$, there exists a upper bound on $\left\|\mathbf{S}-\mathbf{\Phi} \mathbf{C}^{(T)}\right\|_{F}$. This 


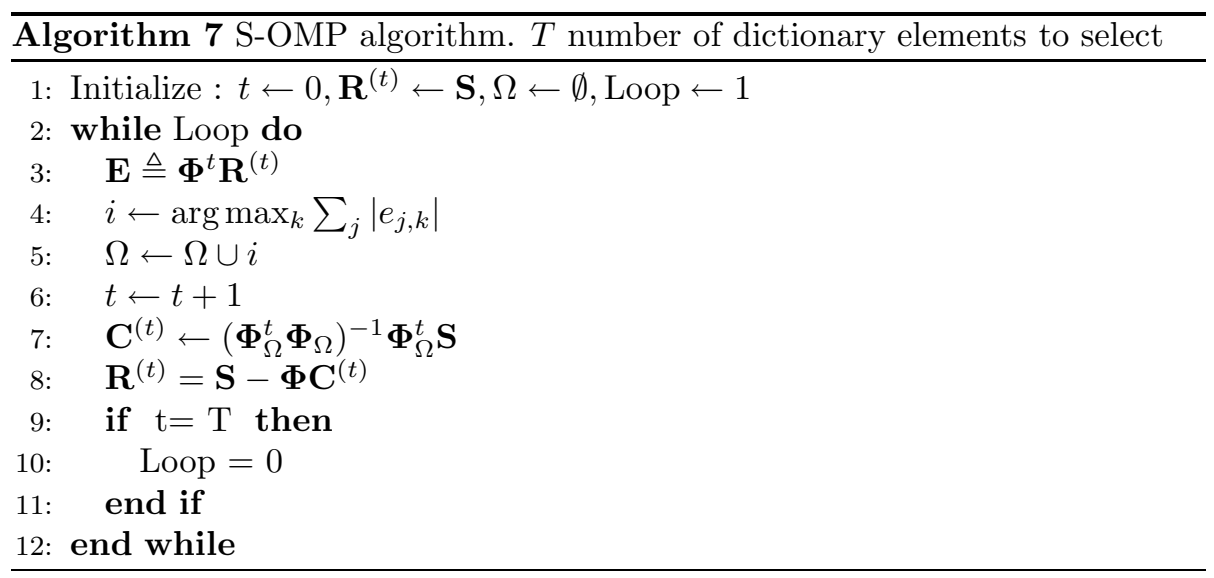

upper bound depends on $\left\|\mathbf{S}-\mathbf{\Phi} \mathbf{C}^{\star}\right\|_{F}$ with $\mathbf{C}^{\star}$ being the solution of problem (2) and multiplicative constant which is a function of $T, L$ and the coherence of $\boldsymbol{\Phi}$ [48]. In this sense, S-OMP can be understood as an algorithm that approximately solves problem (4) with $p=0$.

\section{2. $M-\operatorname{Cos} A m p$}

Very recently, Needell and al. have developed a novel signal reconstruction algorithm known as CosAmp 32]. This algorithm uses ideas from orthogonal matching pursuit but also takes advantage of ideas from the compressive sensing literature. This novel approach provides stronger theoretical guarantees on the quality of signal approximation. The main particularities of CosAmp are the following. Firstly, unlike Orthogonal matching pursuit, CosAmp selects many elements of the dictionary at each iteration, the ones that have the largest correlation with respects to the signal residual. These novel dictionary elements are thus incorporate into the set of current dictionary elements. The other specificity of CosAmp is a pruning dictionary element step after signal estimation. This step is justified by the fact that the signal to approximate is supposed to be sparse.

This CosAmp algorithm have been extended so as to handle signals with specific structures such as block sparse signal [1]. Extension to jointly sparse approximation has also been provided by Duarte et al. [11]. Such an extension of 


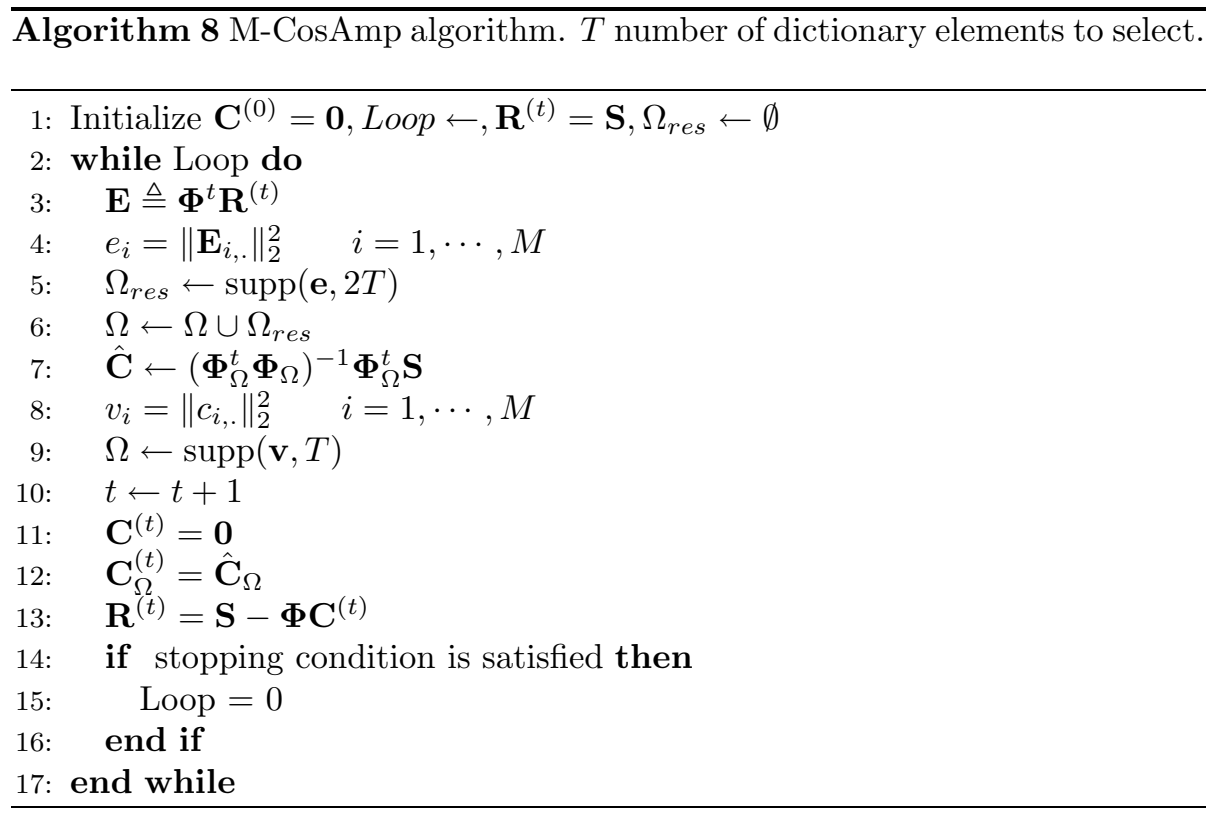

the CosAmp algorithm to simultaneous sparse approximation is detailed in Algorithm 8 Note that lines $4-5$ of the algorithm estimate the dictionary elements which have the largest correlations over all the signals while lines 8-9 prune the set of current dictionary elements so as to keep the matrix $\mathbf{C}$ row-sparse. This algorithm is denoted as M-CosAmp in the experimental section.

\subsection{Sparse Bayesian Learning and Reweighted algorithm}

The approach proposed by Wipf et al. [54], denoted in the sequel as MultipleSparse Bayesian Learning (M-SBL), for solving the sparse simultaneous approximation is somewhat related to the optimization problem in equation (4) but from a very different perspective. Indeed, if we consider that the approaches described in Section 3 are equivalent to a Maximum a Posteriori estimation procedures, then Wipf et al. have explored a Bayesian model which prior encourages sparsity. In this sense, their approach is related to the relevance vector machine of Tipping et al. 46]. Algorithmically, they proposed an empirical bayesian learning approach based on Automatic Relevance Determination (ARD). The 
ARD prior over each row they have introduced is

$$
p\left(c_{i,} ; d_{i}\right)=\mathcal{N}\left(0, d_{i} \mathbf{I}\right) \quad \forall i
$$

where $\mathbf{d}$ is a vector of non-negative hyperparameters that govern the prior variance of each coefficient matrix row. Hence, these hyperparamaters aim at catching the sparsity profile of the approximation. Mathematically, the resulting optimization problem is to minimize according to $\mathbf{d}$ the following cost function :

$$
L \log \left|\Sigma_{t}\right|+\sum_{j=1}^{L} \mathbf{s}_{j}^{t} \Sigma_{t}^{-1} \mathbf{s}_{j}
$$

where $\Sigma_{t}=\sigma^{2} \mathbf{I}+\mathbf{\Phi D} \Phi^{t}, \mathbf{D}=\operatorname{diag}(\mathbf{d})$ and $\sigma^{2}$ a parameter of the algorithm related to the noise level presented in the signals to be approximated. The algorithm is then based on a likelihood maximization which is performed through an Expectation-Minimization approach. Very recently, a very efficient algorithm for solving this problem has been proposed [25]. However, the main drawback of this latter approach is that due to its greedy nature, and as any EM algorithm where the objective function is not convex, the algorithm can be easily stuck in local minima.

Recently, Wipf et al. [51] have proposed some new insights on Automatic Relevance Determination and Sparse Bayesian Learning. They have shown that, for the vector regression case, ARD can be achieved by means of iterative reweighted $\ell_{1}$ minimization. Furthermore, in that paper, they have sketched an extension of such results for matrix regression in which ARD is used for automatically selecting the most relevant covariance components in a dictionary of covariance matrices. Such an extension is more related to learning with multiple kernels in regression as introduced by Girolami et al. 20] or Rakotomamonjy et al. 36] although some connections with simultaneous sparse approximation can be made. Here, we build on the works on Wipf et al. [51] and give all the details about how M-SBL and iterative reweighted M-BP are related. 
Recall that the cost function minimized by the M-SBL of Wipf et al. [54] is

$$
\mathcal{L}(\mathbf{d})=L \log \left|\Sigma_{t}\right|+\sum_{j=1}^{L} \mathbf{s}_{j}^{t} \Sigma_{t}^{-1} \mathbf{s}_{j}
$$

where $\Sigma_{t}=\sigma^{2} \mathbf{I}+\mathbf{\Phi} \mathbf{D} \Phi^{t}$ and $\mathbf{D}=\operatorname{diag}(\mathbf{d})$, with $\mathbf{d}$ being a vector of hyperparameters that govern the prior variance of each coefficient matrix row. Now, let us define $g^{\star}(z)$ as the conjugate function of the concave $\log \left|\Sigma_{t}\right|$. Since, that $\log$ function is concave and continuous on $\mathbb{R}_{+}^{M}$, according to the scaling property of conjugate functions we have [3]

$$
L \cdot \log \left|\Sigma_{t}\right|=\min _{\mathbf{z} \in \mathbb{R}^{M}} \mathbf{z}^{t} \mathbf{d}-L g^{\star}\left(\frac{\mathbf{z}}{L}\right)
$$

Thus, the cost function $\mathcal{L}(\mathbf{d})$ in equation (32) can then be upper-bounded by

$$
\mathcal{L}(\mathbf{d}, \mathbf{z}) \triangleq \mathbf{z}^{t} \mathbf{d}-L g^{\star}\left(\frac{\mathbf{z}}{L}\right)+\sum_{j=1}^{L} \mathbf{s}_{j}^{t} \Sigma_{t}^{-1} \mathbf{s}_{j}
$$

Hence when optimized over all its parameters, $\mathcal{L}(\mathbf{d}, \mathbf{z})$ converges to a local minima or a saddle point of (32). However, for any fixed $\mathbf{d}$, one can optimize over $\mathbf{z}$ and get the tight optimal upper bound. If we denote as $\mathbf{z}^{\star}$ such an optimal $\mathbf{z}$ for any fixed $\mathbf{d}^{\dagger}$, since $L \cdot \log \left|\Sigma_{t}\right|$ is differentiable, we have, according to conjugate function properties, the following closed form of $\mathbf{z}^{\star}$

$$
\mathbf{z}^{\star}=L \cdot \nabla \log \left|\Sigma_{t}\right|\left(\mathbf{d}^{\dagger}\right)=\operatorname{diag}\left(\boldsymbol{\Phi}^{t} \Sigma_{t}^{-1} \boldsymbol{\Phi}\right)
$$

Similarly to what proposed by Wipf et al., Equations (33) and (34) suggest an alternate optimization scheme for minimizing $\mathcal{L}(\mathbf{d}, \mathbf{z})$. Such a scheme would consist, after initialization of $\mathbf{z}$ to some arbitrary vector, in keeping $\mathbf{z}$ fixed and in computing

$$
\mathbf{d}^{\dagger}=\arg \min _{\mathbf{d}} \mathcal{L}_{z}(\mathbf{d}) \triangleq \mathbf{z}^{t} \mathbf{d}+\sum_{j=1}^{L} \mathbf{s}_{j}^{t} \Sigma_{t}^{-1} \mathbf{s}_{j}
$$

then to minimize $\mathcal{L}\left(\mathbf{d}^{\dagger}, \mathbf{z}\right)$ for fixed $\mathbf{d}^{\dagger}$, which can be analytically done according to equation (34). This alternate scheme is then performed until convergence to some $\mathbf{d}^{\star}$. 
Owing to this iterative scheme proposed for solving M-SBL, we can now make clear the connection between M-SBL and an iterative reweighted M-BP according to the following lemma. Again this is an extension to the multiple signals case of a Wipf's lemma.

Lemma 2. The objective function in equation (35) is convex and can be equivalently solved by computing

$$
\mathbf{C}^{\star}=\arg \min _{\mathbf{C}} \mathcal{L}_{z}(\mathbf{C})=\frac{1}{2}\|\mathbf{S}-\mathbf{\Phi} \mathbf{C}\|_{F}^{2}+\sigma^{2} \sum_{i} z_{i}^{1 / 2}\left\|c_{i, \cdot}\right\|
$$

and then by setting

$$
d_{i}=z_{i}^{-1 / 2}\left\|c_{i, .}^{\star}\right\| \quad \forall i
$$

Proof 2. Convexity of the objective function in equation (35) is straightforward since it is just a sum of convex functions [2]. The key point of the proof is based on the equality

$$
\mathbf{s}_{j}^{t} \Sigma_{t}^{-1} \mathbf{s}_{j}=\frac{1}{\sigma^{2}} \min _{c, j}\left\|\mathbf{s}_{j}-\mathbf{\Phi} c_{\cdot, j}\right\|_{2}^{2}+\sum_{i} \frac{c_{i, j}^{2}}{d_{i}}
$$

which proof is given in the appendix. According to this equality, we can upperbound $\mathcal{L}_{z}(\mathbf{d})$ with

$$
\mathcal{L}_{z}(\mathbf{d}, \mathbf{C})=\mathbf{z}^{t} \mathbf{d}+\sum_{j} \frac{1}{\sigma^{2}}\left\|\mathbf{s}_{j}-\mathbf{\Phi} c_{\cdot, j}\right\|_{2}^{2}+\sum_{i, j} \frac{c_{i, j}^{2}}{d_{i}}
$$

The problem of minimizing $\mathcal{L}_{z}(\mathbf{d}, \mathbf{C})$ is smooth and jointly convex in its parameters $\mathbf{C}$ and $\mathbf{d}$ and thus an iterative coordinate-wise optimization scheme (iteratively optimizing over $\mathbf{d}$ with fixed $\mathbf{C}$ and then optimizing over $\mathbf{C}$ with fixed $\mathbf{d}$ ) yields to the global minimum. It is easy to show that for any fixed $\mathbf{C}$, the minimal value of $\mathcal{L}_{z}(\mathbf{d}, \mathbf{C})$ with respects to $\mathbf{d}$ is achieved when

$$
d_{i}=z_{i}^{-1 / 2}\left\|c_{i, \cdot}\right\| \quad \forall i
$$

Plugging these solutions back into [38) and multiplying the the resulting objective function with $\sigma^{2} / 2$ yields to

$$
\mathcal{L}_{z}(\mathbf{C})=\frac{1}{2} \sum_{j}\left\|\mathbf{s}_{j}-\mathbf{\Phi} c_{\cdot, j}\right\|_{2}^{2}+\sigma^{2} \sum_{i} z_{i}^{1 / 2}\left\|c_{i, \cdot}\right\|
$$


Making the relation between $\ell_{2}$ and Frobenius norms concludes the proof.

Minimizing $\mathcal{L}_{z}(\mathbf{C})$ boils down to minimize the M-BP problem with an adaptive penalty $\lambda_{i}=\sigma^{2} \cdot z_{i}^{1 / 2}$ on each row-norm. This latter point makes the alternate optimization scheme based on equation (34) and (35) equivalent to our iterative reweighted M-BP for which weights $z_{i}$ would be given by equation (34).

The impact of this relation between M-SBL and iterative reweighted M$\mathrm{BP}$ is essentially methodological. Indeed, its main advantage is that it turns the original M-SBL optimization problem into a serie of convex optimization problems. In this sense, the iterative reweighted algorithm described here, can again be viewed as an application of MM approach for solving problem (32). Indeed, we are actually iteratively minimizing a proxy function which has been obtained by majorizing each term of equation (32). Owing to this MM point of view, convergence of our iterative algorithm towards a local minimum of equation (32) is guaranteed 24]. Convergence for the single signal case using other arguments has also been shown by Wipf et al. [51]. Note that similarly to M-FOCUSS, the original M-SBL algorithm based on EM approach suffers from presence of fixed points (when $d_{i}=0$ ). Hence, such an algorithm is not guaranteed to converge towards a local minimum of (32). This is then another argument for preferring IrM-BP.

\section{Numerical experiments}

Some computer simulations have been carried out in order to evaluate the algorithms proposed in the above sections. Results that have been obtained from these numerical studies are detailed in this section.

\subsection{Experimental set-up}

In order to quantify the performance of our algorithms and compare them to other approaches, we have used simulated datasets with different redundancy factors $\frac{M}{N}$, number $k$ of active elements and number $L$ of signals to approximate. The dictionary $\boldsymbol{\Phi}$ is based on $M$ vectors sampled from the unit hypersphere 
of $\mathbb{R}^{N}$. The true coefficient matrix $\mathbf{C}^{\star}$ has been obtained as follows. The positions of the $k$ non-zero rows in the matrix are randomly drawn. The nonzero coefficients of $\mathbf{C}^{\star}$ are then drawn from a zero-mean unit variance Gaussian distribution. The signal matrix $\mathbf{S}$ is obtained as in equation (1) with the noise matrix being drawn i.i.d from a zero-mean Gaussian distribution and variance so that the signal-to-noise ratio of each single signal is $10 \mathrm{~dB}$. For a given experiment, when several trials are needed, we only resample the dictionary $\mathbf{\Phi}$ and the additive noise $\mathcal{E}$.

Each algorithm is provided with the signal matrix $\mathbf{S}$ and the dictionary $\mathbf{\Phi}$ and will output an estimate of $\mathbf{C}$. The performance criterion we have considered are the mean-square error between the true and the approximate signals and the sparsity profile of the coefficient matrix that has been recovered. For the latter, we use as a performance criterion the F-measure between the row-support of the true matrix $\mathbf{C}^{\star}$ and the estimate one $\hat{\mathbf{C}}$. In order to take into account numerical precisions, we have overloaded the row support definition as :

$$
\operatorname{rowsupp}(\mathbf{C})=\left\{i \in[1 \cdots M]:\left\|c_{i, \cdot}\right\|<\mu\right\}
$$

where $\mu$ is a threshold coefficient that has been set by default to 0.01 in our experiments. From $\operatorname{rowsupp}(\hat{\mathbf{C}})$ and $\operatorname{rowsupp}\left(\mathbf{C}^{\star}\right)$ respectively the estimated and true sparsity profile, we define :

$$
\text { F-measure }=2 \cdot \frac{\left|\operatorname{rowsupp}(\hat{\mathbf{C}}) \cap \operatorname{rowsupp}\left(\mathbf{C}^{\star}\right)\right|}{|\operatorname{rowsupp}(\hat{\mathbf{C}})|+\left|\operatorname{rowsupp}\left(\mathbf{C}^{\star}\right)\right|} .
$$

Note that the F-measure is equal to 1 when the estimated sparsity profile coincides exactly with the true one.

Regarding the stopping criterion, in the experiments presented below, we have considered convergence of our M-BCD algorithm when the optimality conditions given in equation (77) are satisfied up to a tolerance of 0.001 and when all matrix coefficient $c_{i, j}$ variations are smaller than 0.001 . This latter condition has also been used as a stopping criterion for the Landweber iteration, M-EM, IrM-BP, M-FOCUSS and M-SBL algorithms. When the annealing approach is 

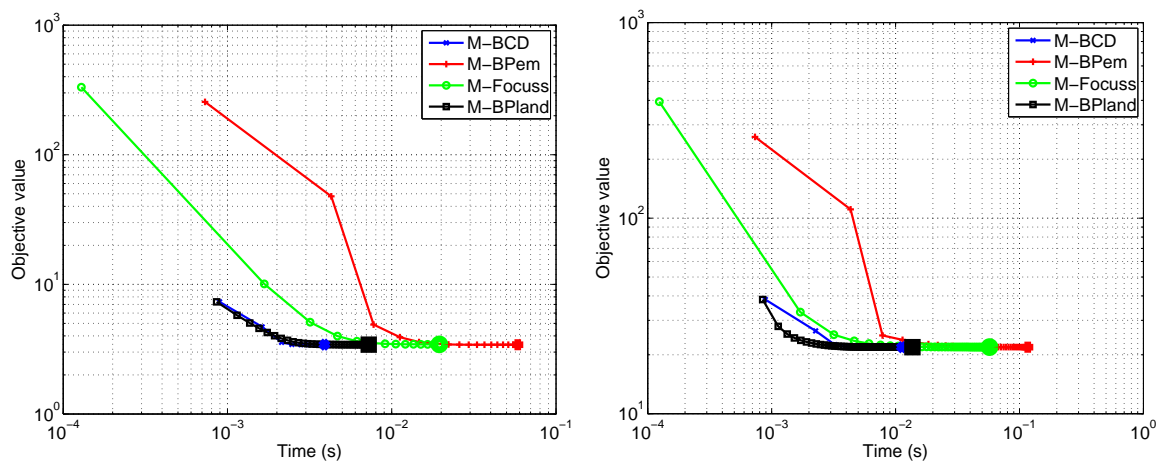

Figure 1: Examples of objective value evolution with respects to computational time. Here we have, $M=128, N=64, L=3$. The number of active elements is : left) $k=5$. right) $k=32$. For each curve, the large point corresponds to the objective value at convergence.

in play for IrM-BP and M-FOCUSS, the annealing loop is also stopped under the same condition e.g $\max _{i, j}\left|C_{i, j}^{(t+1)}-C_{i, j}^{(t)}\right|<0.001$ where $(t)$ and $(t+1)$ denotes two consecutive annealing solution.

\subsection{Comparing $\ell_{1}-\ell_{2} M-B P$ problem solvers}

In this first experiment, we have compared different algorithms which solves the M-BP problem with $p=1$ and $q=2$. Besides our M-BCD and M-EM algorithms, we have also used the M-FOCUSS of Cotter et al. [8] and the approach of Fornasier et al. 17] based on Landweber iterations and denoted in the sequel as M-BPLand. Note that for M-FOCUSS, we have modified the genuine algorithm by introducing a $\varepsilon$ parameter, set to 0.001 , which helps in avoiding a row-norm of $\mathbf{C}$ to be permanently at 0 . For all compared problems, regularization parameter $\lambda$ has been so as to make the problem equivalent. In this experiment, we have set $\lambda=\frac{1}{5} \max _{i}\left\|\phi_{i}^{t} \mathbf{S}\right\|_{2}$ for problem (6).

Figure 1 shows two examples of how the objective value of the different algorithms evolves with respects to computational time. We can note that the two iterative reweighted least-square algorithms (M-EM and M-FOCUSS) are the most computationally demanding. Furthermore, we also see that the Landweber iteration approach of Fornasier et al. quickly reduces its objective value but compared to our M-BCD method, it needs more time before properly converging. Table 1 summarizes more accurately the difference between the 
Table 1: Summary of M-BP solvers comparison. Comparisons have been carried out for two values of $k$, the number of active elements in the dictionary and have been averaged over 100 trials. Comparison measures are the time needed before convergence, the difference in objective value and the largest coefficient matrix difference. For the two latter measure, the baseline algorithm is considered to be the M-BCD one.

\begin{tabular}{|c|c|c|c|}
\hline \multicolumn{4}{|c|}{$\mathrm{k}=5$} \\
\hline$\overline{\mathrm{M}-\mathrm{BCD}}$ & $44.2 \pm 2.7$ & - & $\begin{array}{c}- \\
-\end{array}$ \\
\hline M-EM & $57.2 \pm 11.7$ & $2.6 \pm 2.2$ & $0.8 \pm 1.0$ \\
\hline M-Focuss & $24.1 \pm 4.2$ & $3.6 \pm 1.8$ & $16.6 \pm 1.0$ \\
\hline M-BPLand & $7.5 \pm 1.3$ & $5.3 \pm 1.1$ & $0.04 \pm 1.1$ \\
\hline & Time (ms) & $\begin{array}{l}\mathbf{k}=\mathbf{3 2} \\
\Delta \text { ObjVal }\left(10^{-3}\right)\end{array}$ & $\|\Delta \mathbf{C}\|_{\infty}\left(10^{-3}\right)$ \\
\hline$\overline{\mathrm{M}-\mathrm{BCD}}$ & $111.5 \pm 2.87$ & 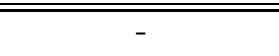 & 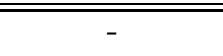 \\
\hline M-EM & $130.9 \pm 17.0$ & $23.1 \pm 5.9$ & $15.4 \pm 8.0$ \\
\hline M-Focuss & $60.0 \pm 6.2$ & $28.8 \pm 5.8$ & $38.8 \pm 5.4$ \\
\hline M-BPLand & $14.1 \pm 2.7$ & $16.9 \pm 3.8$ & $0.41 \pm 3.8$ \\
\hline
\end{tabular}

four algorithms. These results have been averaged over 100 trials and consider two values of $k$. As comparison criteria, we have considered the computational time before convergence, the difference (compared to the M-BCD algorithm) in objective values and the maximal absolute difference in the coefficient matrix $c_{i, j}$. The table clearly shows that the M-BCD algorithm is clearly faster than M-BPLand (especially when signals to approximate are highly sparse) and the two iterative reweighted least-square approaches. We can also note from the table that, although M-FOCUSS and our M-EM are not provided with a formal convergence proof, these two algorithms seem to empirically converge to the problem global minimum.

\subsection{Computational performances}

We have also empirically assessed the computational complexity of our algorithms (we used $s=0.2$, thus $q=\frac{5}{3}$ for M-EM and $r=1$ for IrM-BP). We varied one of the different parameters (dictionary size $M$, signal dimensionality $N$ ) while keeping the others fixed. All matrices $\mathbf{\Phi}, \mathbf{C}$ and $\mathbf{S}$ are created as described above. Experiments have been run on a Pentium D-3 GHz with 
4 GB of RAM using Matlab code. The results in Figure 2 averaged over 20 trials, show the computational complexity of the different algorithms for different experimental settings. Note that we have also experimented on the M-SBL computational performances owing to the code of Wipf et al. [54] and have implemented the M-FOCUSS of Cotter et al. [8], the CosAmp block-sparse approach of Baraniuk et al. 1] and the Landweber iteration method of Fornasier et al. 17 4]. All algorithms need one hyperparameter to be set, for M-SBL and CosAmp, we were able to choose the optimal one since the hyperparameter respectively depends on a known noise level and a known number of active elements in the dictionary. For other algorithms, we have reported the computational complexity for the $\lambda$ that yields to the best sparsity recovery. Note that our aim here is not give an exact comparison of computational complexity of the algorithms but just to give an order of magnitude of these complexities. Indeed, accurate comparisons are difficult since the different algorithms do not solve the same problem and do not use the same stopping criterion.

We can remark in Figure 2 that with respects to the dictionary size, all algorithms present an empirical exponent between 1.3 and 2.7. Interestingly, we have theoretically evaluate the complexity of the M-BCD algorithm as quadratic whereas we measure a sub-quadratic complexity. We suppose that this happens because at each iteration, only the non-optimal $c_{i}$,'s are updated and thus the number of updates drastically reduces along iterations. We can note that among all approaches that solve the $\ell_{1}-\ell_{q}$ problem (left plots), M-BCD, Landweber iteration approach and M-CosAmp have similar complexity with a slight advantage to M-BCD for large dictionary size. However, we have to note that the M-CosAmp algorithm sometimes suffers from lack of convergence and thus stop only when the maximal number of allowed iterations is reached. This is the reason why for large dictionary size M-CosAmp is computationally expensive. However, for small and medium dictionary size, M-CosAmp is slighly more efficient than M-BCD. When considering the algorithms that solve the $\ell_{p}-\ell_{2}$

\footnotetext{
${ }^{4}$ All the implementations are included in the toolbox.
} 
problem (right plots), they all have similar complexity, with a slightly better constant for IrM-BP while M-SBL seems to be the most demanding algorithm.

Bottom plots of Figure 2 depicts the complexity dependency of all algorithms with respects to signal dimension $N$. Interestingly, the results show that except for M-SBL and M-FOCUSS algorithms, all algorithms do not suffer from the signal dimension increase. We assume that this is due to the fact that as dimension increases, the approximation problem becomes easier and thus faster convergence of those algorithms occurs.

\subsection{Comparing performances}

The objective of the next empirical study is to compare the performances of the algorithms we surveyed : M-SBL, M-CosAmp, Landweber iterations, SOMP, M-FOCUSS with an annealing decreasing of $\varepsilon$, the M-BCD algorithm and the IrM-BP approach with two values of $p$ and an annealing decrease of $\varepsilon$.

The baseline experimental context is $M=128, N=64, k=10$ and $L=$ 3. For this experiment, we have considered an agnostic context with no prior knowledge about the noise level being available. Hence, for all models, we have performed model selection (either for selecting $\lambda$, the noise level $\sigma$ for M-SBL or the number of elements for M-CosAmp and S-OMP). Model selection procedure is the following. Training signals $\mathbf{S}$ are randomly splitted in two parts of $N / 2$ samples. Each algorithm is then trained on one part of the signal and the mean-square error of the resulting model is evaluated on the second part. This splitting and training is run 5 times and the hyperparameter yielding to the minimal averaged mean-square error is considered as optimal. Each method is then run on the full signals with that parameter. Performances, averaged over 50 trials of all methods have been evaluated according to the F-measure and a mean-square error computed on 10000 samples.

Figure 3 shows, from top to bottom, these performances when $k$ increases from 2 to 40, when $M$ goes from 64 to 256 and when $L=2, \cdots, 7$. When varying $k$, we can note that across the range of variation, our IrM-BP method with $p=0$ is competitive compared to all other approaches both with respects to 
the F-measure and the mean-square error criterion. When $k$ increases, IrM-BP and M-FOCUSS with $p=0.5$ perform also very good. This may be explained by the fact that as $k$ increases, the optimal solution becomes less and less sparse thus the need for a less aggressive penalty. CosAmp and S-OMP are very competitive for small $k$ but as soon as the latter increases these two methods are not able anymore to recover a "reasonable" sparsity pattern. Interestingly, we remark that M-SBL yields to a poor sparsity recovery measure while the resulting model achieves good mean-square error. A reason for this is that the model selection procedure tends to under-estimate the noise level and thus it leads to a model which keeps many spurious dictionary elements as illustrated in Figure 4 and detailed in the sequel. From Figure 3 we can also notice that the two M-BP solvers, our M-BCD and the Landweber iteration approach perform poorly compared to other methods. However, the Fornasier's method seems to be less sensitive to noise and model selection since it provides a better sparsity pattern recovery. It is worth noting that M-SBL and these two latter methods always correctly select all the true dictionary elements but they also have the tendency to include other spurious ones.

In the middle and bottom plots, similar behavior as above can be highlighted. M-CosAmp yields to very good sparsity recovery while the resulting mean-square error is rather poor. Again our IrM-BP with $p=0$ yields the best mean-square error while providing a good sparsity pattern recovery. M-SBL and M-BCD keeps too many spurious dictionary elements. All other methods provide in-between performances both in term of $\mathrm{F}$-measure and mean-square error.

Figure 4 illustrates the behaviour of M-CosAmp, M-SBL and the IrM-BP with $p=0$ for two different experimental situations. On the left plot, we have a case where on one hand, M-CosAmp misses to recover the first active dictionary element yielding thus to high mean-square error. On the other hand, M-SBL achieves lower mean-square error while keeping few spurious dictionary elements in the model. In the meantime, IrM-BP recovers perfectly the sparsity pattern and yields to low mean-square error. In the right plot, we have another case 
where M-CosAmp achieves perfect sparsity recovery but provides a model with higher mean-square error than IrM-BP.

In most of the experimental situations presented here, M-CosAmp and the IrM-BP seems to be the two algorithms that perform the best, with however a slight advantage for the IrM-BP. These two methods are actually related since both approaches solve a simultaneous sparse approximation with a $J_{0,2}(\mathbf{C})$ penalty. The main difference lies in the algorithms since our IrM-BP owing to the $\varepsilon$ term provides a smooth approximation of the $\ell_{0}$ quasi-norm whereas MCosAmp directly solves the approximation problem with the $J_{0,2}(\mathbf{C})$ penalty.

To summarize, we suggest the following rule : in applications where computational complexities are critical and where signals to be approximated are highly sparse, M-CosAmp is to be preferred. In other situations, IrM-BP should be used.

\section{Conclusions}

This paper aimed at surveying and comparing different simultaneous sparse signal approximation algorithms available in the statistical learning and signal processing communities.

These algorithms usually solve a relaxed version of an intractable problem where the relaxation is based on sparsity-inducing mixed norm on the coefficient approximation. In the first part of the paper, we have described several algorithms which addresses the most common SSA problem which is a convex problem with a $\ell_{1}-\ell_{2}$ penalty function. For other choices of penalty, one usually considers iterative reweighted algorithms. These algorithms have been detailed in a second part of the paper. We have also brought our attention on greedy algorithms such as S-OMP or M-CosAmp since they have both have the abilities to be very efficient and to provide theoretical guarantees on the quality of their solutions.

The last part of the paper is devoted to experimental comparisons of the different algorithms we surveyed. The lesson learned from these comparisons is 
that the two algorithms that seem to be the best performing either in terms of efficiency, sparsity recovery or low mean-square error are the M-CosAmp or an iterative reweighted $\ell_{1}$ approach.

\section{Appendix}

\section{1. $J_{1,2}(\mathbf{C})$ subdifferential}

By definition, a matrix $\mathbf{G}$ lies in $\partial J_{1,2}(\mathbf{B})$ if and only if for every matrix $\mathbf{Z}$, we have

$$
J_{1,2}(\mathbf{Z}) \geq J_{1,2}(\mathbf{B})+\langle\mathbf{Z}-\mathbf{B}, \mathbf{G}\rangle_{F}
$$

If we expand this equation we have the following equivalent expression

$$
\sum_{i}\left\|z_{i, \cdot}\right\|_{2} \geq \sum_{i}\left\|b_{i, \cdot}\right\|_{2}+\sum_{i}\left\langle z_{i, \cdot}-b_{i, \cdot,} g_{i, \cdot}\right\rangle
$$

From this latter equation, we understand that, since both $J_{1,2}$ and the Frobenius inner product are row-separable, a matrix $\mathbf{G} \in \partial J_{1,2}(\mathbf{B})$ if and only if each row

of $\mathbf{G}$ belongs to the subdifferential of the $\ell_{2}$ norm of the corresponding row of B.

Indeed, suppose that $\mathbf{G}$ is so that any row of $\mathbf{G}$ belongs to the subdifferential of the $\ell_{2}$ norm of the corresponding row of $\mathbf{B}$. We thus have for any row $i$

$$
\forall \mathbf{z}, \quad\|\mathbf{z}\|_{2} \geq\left\|b_{i, \cdot}\right\|_{2}+\left\langle z-b_{i, .}, g_{i, \cdot}\right\rangle
$$

A summation over all the rows then proves that $\mathbf{G}$ satisfies equation (41) and thus belongs to the subdifferential of $J_{1,2}(\mathbf{B})$.

Now, let us show that a matrix $\mathbf{G}$ for which there exists a row that does not belong to the subdifferential of the $\ell_{2}$ norm of the corresponding row of $\mathbf{B}$ can

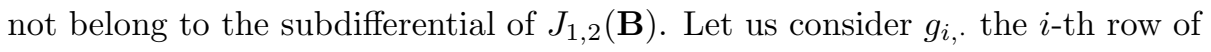
$\mathbf{G}$, since we have supposed that $g_{i,} \notin \partial\left\|b_{i,} \cdot\right\|_{2}$, the following equation holds

$$
\exists \mathbf{z}_{0} \text { st. } \quad\left\|\mathbf{z}_{0}\right\|_{2}<\left\|b_{i, .}\right\|_{2}+\left\langle\mathbf{z}-b_{i, .}, g_{i}\right\rangle
$$

Now let us construct $\mathbf{Z}$ so that $\mathbf{Z}=\mathbf{B}$ except for the $i$-th row where $z_{i,}=\mathbf{z}_{0}$. Then it is easy to show that this matrix $\mathbf{Z}$ does not satisfy equation (41), which 
means that $\mathbf{G}$ does not belong to $\partial J_{1,2}(\mathbf{B})$. In conclusion, we get $\partial J_{1,2}(\mathbf{B})$ by applying the $\ell_{2}$ norm subdifferential to each row of $\mathbf{B}$. And it is well known 2] that

$$
\partial\|\mathbf{b}\|_{2}=\left\{\begin{array}{lr}
\left\{\mathbf{g} \in \mathbb{R}^{L}:\|\mathbf{g}\|_{2} \leq 1\right\} & \text { if } \mathbf{b}=\mathbf{0} \\
\frac{\mathbf{b}}{\|\mathbf{b}\|_{2}} & \text { otherwise }
\end{array}\right.
$$

\subsection{Proof of Lemma 2}

We aim at proving that

$$
\min _{\mathbf{d}}\left\{\sum_{t, k} \frac{\left|a_{t, k}\right|^{2}}{d_{t, k}}: d_{t, k} \geq 0, \sum_{k}\left(\sum_{t} d_{t, k}^{1 / s}\right)^{\frac{s}{r+s}} \leq 1\right\}=\left(\sum_{k}\left(\sum_{t}\left|a_{t, k}\right|^{q}\right)^{\frac{p}{q}}\right)^{\frac{2}{p}}
$$

where $q=\frac{2}{s+1}$ and $p=\frac{2}{s+r+1}$. The proof proceeds by writing the Lagrangian of the optimization problem :

$$
\mathcal{L}=\sum_{t, k} \frac{\left|a_{t, k}\right|^{2}}{d_{t, k}}+\lambda\left(\sum_{k}\left(\sum_{t} d_{t, k}^{1 / s}\right)^{\frac{s}{r+s}}-1\right)-\sum_{t, k} \nu_{t, k} d_{t, k}
$$

where $\lambda$ and $\left\{\nu_{t, k}\right\}$ are the Lagrangian multipliers associated to the inequality constraint and the positivity constraints on $d_{t, k}$. By deriving the first-order optimality conditions, we get :

$$
\frac{\partial \mathcal{L}}{\partial d_{m, n}}=-\frac{\left|a_{m, n}\right|^{2}}{d_{m, n}^{2}}-\nu_{m, n}+\frac{\lambda s}{r+s}\left(\sum_{t} d_{t, n}^{1 / s}\right)^{\frac{-r}{r+s}} \cdot \frac{1}{s} \cdot d_{m, n}^{\frac{1-s}{s}}
$$

According to these optimality conditions, at a stationary point, we have either $d_{m, n}=0$ or

$$
d_{m, n}=\left(\frac{\lambda}{r+s}\right)^{-s /(s+1)}\left|a_{m, n}\right|^{2 s /(s+1)}\left(\sum_{t} d_{t, n}^{1 / s}\right)^{r s /[(r+s)(s+1)]}
$$

Then, we can derive

$$
\left.\left(\sum_{m} d_{m, n}^{1 / s}\right)\right)^{(s+1)}=\left(\frac{r+s}{\lambda}\right)\left(\sum_{m}\left|a_{m, n}\right|^{2 /(s+1)}\right)^{s+1}\left(\sum_{m} d_{m, n}^{1 / s}\right)^{r /(r+s)}
$$


and thus

$$
\left.\left(\sum_{m} d_{m, n}^{1 / s}\right)\right)^{s}=\left(\frac{r+s}{\lambda}\left(\sum_{m}\left|a_{m, n}\right|^{2 /(s+1)}\right)^{s+1}\right)^{(r+s) /(r+s+1)}
$$

As $\lambda \neq 0$, the inequality on the mixed-norm on $d_{t, k}$ becomes an equality. Hence, after powering each side of Equation (46) to $1 /(r+s)$ and summing each side over $n$, we have :

$$
\frac{\lambda}{r+s}=\left(\sum_{n} g_{n}^{(s+1) /(r+s+1)}\right)^{r+s+1}
$$

where $g_{n}=\sum_{m}\left|a_{m, n}\right|^{2 /(s+1)}$. Then, plugging equations (47) and (46) into (44) gives the desired result :

$$
d_{m, n}=\frac{\left|a_{m, n}\right|^{\frac{2 s}{s+1}} g_{n}^{\frac{r}{s+r+1}}}{\left(\sum_{n} g_{n}^{\frac{s+1}{s+r+1}}\right)^{r+s}}
$$

\subsection{Proof of equation (37)}

We want to show that at optimality which occurs at $\mathbf{C}^{\star}$, we have

$$
\mathbf{s}_{j}^{t} \Sigma_{t}^{-1} \mathbf{s}_{j}=\frac{1}{\sigma^{2}} \mathbf{s}_{j}^{t}\left(\mathbf{s}_{j}-\mathbf{\Phi} \mathbf{C}^{\star}\right)
$$

which is equivalent, after factorizing with $\mathbf{s}^{t}$, to show that

$$
\sigma^{2} \mathbf{s}_{j}=\Sigma_{t} \mathbf{s}_{j}-\Sigma_{t} \boldsymbol{\Phi} \mathbf{C}^{\star}
$$

This last equation can be proved using simple algebra

$$
\begin{aligned}
\Sigma_{t} \mathbf{s}_{j}-\Sigma_{t} \boldsymbol{\Phi} \mathbf{C} & =\sigma^{2} \mathbf{s}_{j}+\boldsymbol{\Phi} \mathbf{D} \boldsymbol{\Phi}^{t} \mathbf{s}-\left(\sigma^{2} I+\boldsymbol{\Phi} D \boldsymbol{\Phi}^{t}\right) \boldsymbol{\Phi} \mathbf{C}^{\star} \\
& =\sigma^{2} \mathbf{s}_{j}+\boldsymbol{\Phi} \mathbf{D} \boldsymbol{\Phi}^{t} \mathbf{s}-\boldsymbol{\Phi}\left(\sigma^{2} I+D \boldsymbol{\Phi}^{t} \boldsymbol{\Phi}\right) \mathbf{C}^{\star} \\
& =\sigma^{2} \mathbf{s}_{j}+\boldsymbol{\Phi} \mathbf{D} \boldsymbol{\Phi}^{t} \mathbf{s}-\boldsymbol{\Phi} \mathbf{D} \boldsymbol{\Phi}^{t} \mathbf{s} \\
& =\sigma^{2} \mathbf{s}_{j}
\end{aligned}
$$

\section{References}

[1] Baraniuk, R. G., Cevher, V., Duarte, M., Hegde, C., 2009. Model-based compressive sensing. IEEE Transactions on Information Theory to appear. 
[2] Bertsekas, D., Nedic, A., Ozdaglar, A., 2003. Convex Analysis and Optimization. Athena Scientific.

[3] Boyd, S., Vandenberghe, L., 2004. Convex Optimization. Cambridge University Press.

[4] Candès, E., Wakin, M., Boyd, S., 2008. Enhancing sparsity by reweighted $\ell_{1}$ minimization. J. Fourier Analysis and Applications 14, 877-905.

[5] Chartrand, R., Yin, W., 2008. Iteratively reweighted algorithms for compressive sensing. In: 33rd International Conference on Acoustics, Speech, and Signal Processing (ICASSP).

[6] Chen, J., Huo, X., 2005. Sparse representations for multiple measurements vectors $(\mathrm{mmv})$ in an overcomplete dictionary. In: Proc IEEE Int. Conf Acoustics, Speech Signal Processing. Vol. 4. pp. 257-260.

[7] Chen, S., Donoho, D., Saunders, M., 1999. Atomic decomposition by basis pursuit. SIAM Journal Scientific Comput. 20 (1), 33-61.

[8] Cotter, S., Rao, B., Engan, K., Kreutz-Delgado, K., 2005. Sparse solutions to linear inverse problems with multiple measurement vectors. IEEE Transactions on Signal Processing 53 (7), 2477-2488.

[9] Daubechies, I., Defrise, M., Mol, C. D., 2004. An iterative thresholding algorithm for linear inverse problems with a sparsity constraint. Communication Pure Applied Mathematics 57, 1413-1541.

[10] Daubechies, I., DeVore, R., Fornasier, M., Gunturk, S., 2009. Iteratively reweighted least squares minimization for sparse recovery. Commun. Pure Appl. Math to appear.

[11] Duarte, M., Cevhler, V., Baraniuk, R., 2009. Model-based compressive sensing for signal ensembles. In: Proceeding of the 47th Allerton Conference on Communication, Control and Computing. 
[12] Elad, M., 2006. Why simple shrinkage is still relevant for redundant representations? IEEE Trans. on Information Theory 52 (12), 5559-5569.

[13] Eldar, Y., Kuppinger, P., Blocskei, H., 2009. Compressed sensing of blocksparse signals : Uncertainty relations and efficient recovery. IEEE Trans. Signal Processing to appear.

[14] Eldar, Y., Mishali, M., To appear. Robust recovery of signals from a structured union of subspaces. IEEE Trans. Information Theory.

[15] Fan, J., Li, R., 2001. Variable selection via nonconcave penalized likelihood and its oracle properties. Journal of the American Statistical Association 96 (456), 1348-1360.

[16] Figueiredo, M., Bioucas-Dias, J., R., N., 2007. Majorization-minimization algorithms for wavelet-based image for restoration. IEEE Trans. on Image Processing 16 (12), 2980-2991.

[17] Fornasier, M., Rauhut, H., 2008. Recovery algorithms for vector valued data with joint sparsity constraints. SIAM Journal of Numerical Analysis $46(2), 577-613$.

[18] Foucart, S., Lai, M.-J., 2009. Sparsest solutions of underdetermined linear systems via $\ell_{q}$-minimization for $0<q \leq 1$. Applied and Computational Harmonic Analysis 26 (3), 395-407.

[19] Friedman, J., Hastie, T., Hölling, H., Tibshirani, R., 2007. Pathwise coordinate optimization. The Annals of Applied Statistics 1 (2), 302-332.

[20] Girolami, M., Rogers, S., 2005. Hierarchic bayesian models for kernel learning. In: Proc. of 22nd International Conference on Machine Learning. pp. $241-248$.

[21] Gorodnitsky, I., George, J., Rao, B., 1995. Neuromagnetic source imaging with FOCUSS : a recursive weighted minimum norm algorithm. J. Electroencephalogr. Clin. Neurophysiol. 95 (4), 231-251. 
[22] Grandvalet, Y., 1998. Least absolute shrinkage is equivalent to quadratic penalization. In: Niklasson, L., Bodén, M., Ziemske, T. (Eds.), ICANN'98. Vol. 1 of Perspectives in Neural Computing. Springer, pp. 201-206.

[23] Huang, J., Ma, S., Xie, H., Zhang, C., 2009. A group bridge approach for variable selection. Biometrika 96 (2), 339-355.

[24] Hunter, D., Lange, K., 2004. A tutorial on MM algorithms. The American Statistician 58, 30-37.

[25] Ji, S., Dunson, D., Carin, L., 2008. Multi-task compressive sensing. IEEE Trans. Signal Processing 57 (1), 92-106.

[26] Kim, Y., Kim, J., Kim, Y., 2006. blockwise sparse regression. Statistica Sinica 16, 375-290.

[27] Liu, H., Zhang, J., 2009. On the estimation and variable selection consistency of the sum of q-norm regularized regression. Tech. rep., Department of Statistics, Carnegie Mellon University.

[28] Luo, Z., Gaspar, M., Liu, J., Swami, A., 2006. Distributed signal processing in sensor networks. IEEE Signal Processing magazine 23 (4), 14-15.

[29] Malioutov, D., Cetin, M., Willsky, A., 2005. Sparse signal reconstruction perspective for source localization with sensor arrays. IEEE Trans. Signal Processing 53 (8), 3010-3022.

[30] Mallat, S., Zhang, Z., 1993. Matching pursuit with time-frequency dictionaries. IEEE Trans Signal Processing 41 (12), 3397-3415.

[31] Mishali, M., Eldar, Y., 2008. Reduce and boost : Recovering arbitrary sets of jointly sparse vectors. IEEE Trans. On Signal Processing 56 (10), $4692-4702$.

[32] Needell, D., Tropp, J., 2009. Cosamp: Iterative signal recovery from incomplete and inaccurate samples. Applied and Computational Harmonic Analysis 26 (3), 301-321. 
[33] Obozinski, G., Taskar, B., Jordan, M., 2009. Joint covariate selection and joint subspace selection for multiple classification problems. Statistics and Computing to appear.

[34] Pati, Y., Rezaiifar, R., Krishnaprasad, P., 1993. Orthogonal matching pursuit : Recursive function approximation with applications to wavelet decomposition. In: Proc. of the 27th Annual Asilomar Conference on Signals, Systems and Computers.

[35] Phillips, C., Mattout, J., Rugg, M., Maquet, P., Friston, K., 2005. An empirical Bayesian solution to the source reconstruction problem in EEG. NeuroImage 24, 997-1011.

[36] Rakotomamonjy, A., Bach, F., Grandvalet, Y., Canu, S., 2008. SimpleMKL. Journal of Machine Learning Research 9, 2491-2521.

[37] Rao, B., Engan, K., Cotter, S., Palmer, J., Kreutz-Delgado, K., 2003. Subset selection in noise based on diversity measure minimization. IEEE Trans. Signal Processing 51 (3), 760-770.

[38] Saab, R., Chartrand, R., Özgür Yilmaz, 2008. Stable sparse approximations via nonconvex optimization. In: 33rd International Conference on Acoustics, Speech, and Signal Processing (ICASSP).

[39] Sardy, S., Bruce, A., Tseng, P., 2000. Block coordinate relaxation methods for non-parametric wavelet denoising. Journal of Computational and Graphical Statistics 9 (2), 361-379.

[40] Simila, T., 2007. Majorize-minimize algorithm for multiresponse sparse regression. In: IEEE International Conference on Acoustics, Speech, and Signal Processing (ICASSP). pp. 553-556.

[41] Simila, T., Tikka, J., 2007. Input selection and shrinkage in multiresponse linear regression. Computational Statistics and Data analysis 52 (1), 406422. 
[42] Stojnic, M., Parvaresh, F., Hassibi, B., 2008. On the reconstruction of block-sparse signals with an optimal number of measurements. Tech. Rep. ArXiv 0804.0041v1, California Instite of Technology.

[43] Sturm, J., 1999. Using SeDuMi 1.02 a Matlab toolbox for optimization over symmetric codes. Optimization Methods and Software 11 (12), 625-653.

[44] Sun, L., Liu, J., Chen, J., Ye, J., 2009. Efficient recovery of jointly sparse vectors. In: Advances in Neural Information Processing Systems 23.

[45] Tibshirani, R., 1996. Regression shrinkage and selection via the lasso. Journal of the Royal Statistical Society 46, 267-288.

[46] Tipping, M., 2001. Sparse Bayesian Learning and the Relevance Vector Machine. Journal of Machine Learning Research 1, 211-244.

[47] Tropp, J., Gilbert, A., 2007. Signal recovery from random measurements via orthogonal matching pursuit. IEEE Trans. Information Theory 53 (12), $4655-4666$.

[48] Tropp, J., Gilbert, A., Strauss, M., 2006. Algorithms for simultaneous sparse approximation. part I: Greedy pursuit. Journal of Signal Processing $86,572-588$.

[49] Tseng, P., 2001. Convergence of block coordinate descent method for nondifferentiable minimization. Journal of Optimization Theory and Application $109,475-494$.

[50] van den Berg, E., Schmidt, M., Friedlander, M., Murphy, K., 2008. Group sparsity via linear-time projection. Tech. Rep. TR-2008-09, University of British Columbia, Department of Computer Science.

[51] Wipf, D., Nagarajan, S., 2008. A new view of automatic relevance determination,. In: Advances in Neural Information Processing Systems. Vol. 20. MIT Press, Cambridge, MA. 
[52] Wipf, D., Nagarajan, S., 2010. Iterative reweighted $\ell_{1}$ and $\ell_{2}$ methods for finding sparse solutions. Journal of Selected Topics in Signal Processing, Special Issue on Compressive Sensing to appear.

[53] Wipf, D., Owen, J., Attias, H., Sekihara, K., Nagarajan, S., 2010. Robust bayesian estimation of the location orientation and time course of multiple correlated neural sources using meg. NeuroImage 49 (1), 641-655.

[54] Wipf, D., Rao, B., Jul. 2007. An empirical bayesian strategy for solving the simultaneous sparse approximation problem. IEEE Trans on Signal Processing 55 (7), 3704-3716.

[55] Yuan, M., Lin, Y., 2006. Model selection and estimation in regression with grouped variables. Journal of Royal Statisctics Society B 68, 49-67.

[56] Zhou, H., Hastie, T., 2005. Regularization and variable selection via the elastic net. Journal of the Royal Statistics Society Ser. B 67, 301-320.

[57] Zou, H., 2006. The adaptive lasso and its oracle properties. Journal of the American Statistical Association 101 (476), 1418-1429.

[58] Zou, H., Li, R., 2008. One-step sparse estimates in nonconcave penalized likelihood models. The Annals of Statistics 36 (4), 1509-1533. 

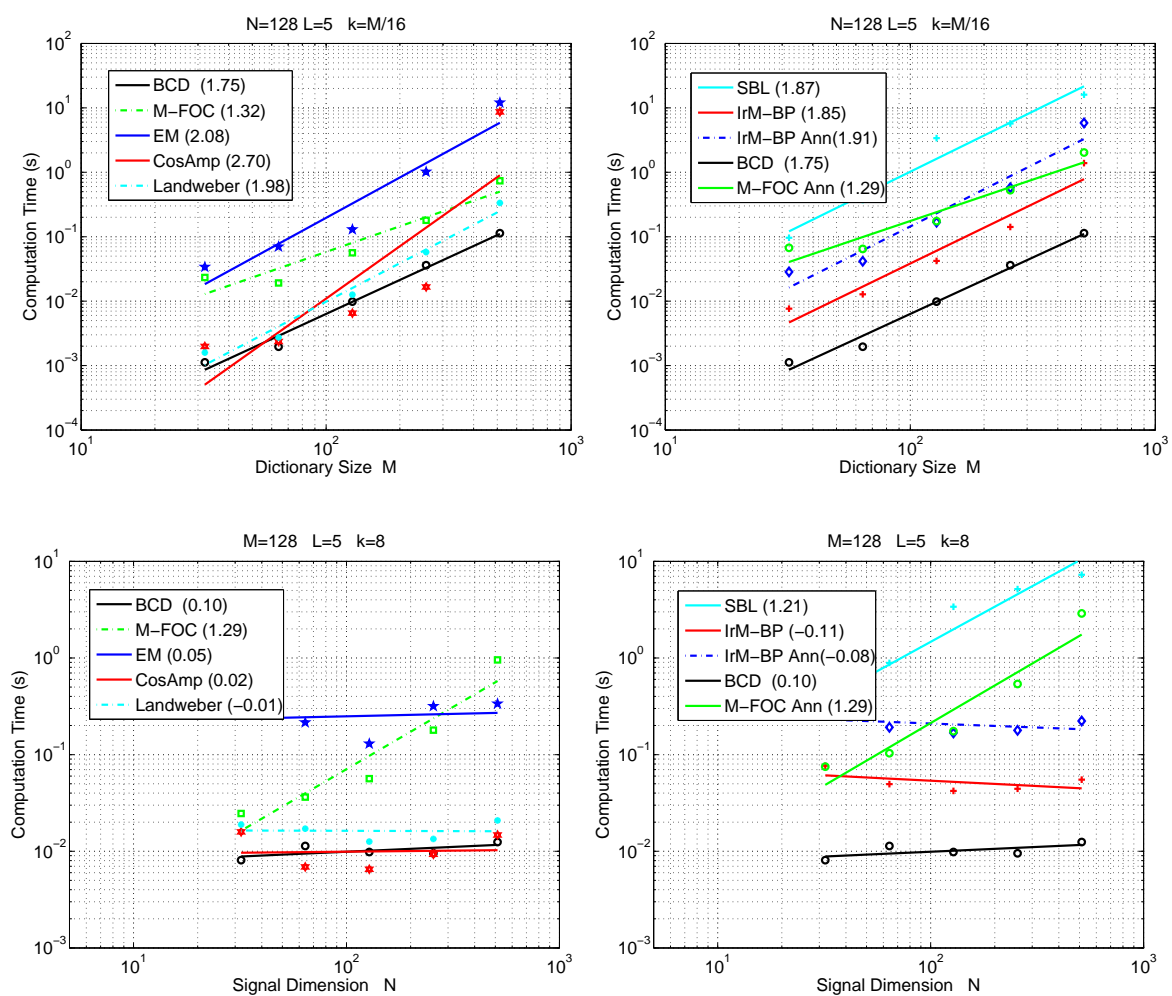

Figure 2: Estimating the empirical exponent, given in parenthesis, of the computational complexity of different algorithms (M-BCD, IrM-BP, M-SBL, M-FOCUSS, CosAmp, Landweber iterations). The top plots give the computation time of the algorithms with respects to the dictionary size. The bottom plots respectively depict the computational complexity with respects to the signal dimensionality. For a sake of readability, we have separated the algorithms in two groups :(left) the ones that solve $\ell_{1}-\ell_{q}$ problem. (right) the ones that solve $\ell_{p}-\ell_{2}$ problem (M-BCD result provided for baseline comparison). The "IrM-BP Ann" and "M-FOC Ann" refers to the Ir-MBP and M-FOCUSS algorithm using an annealing approach for iteratively decreasing $\varepsilon$ as described in Algorithm (??). 

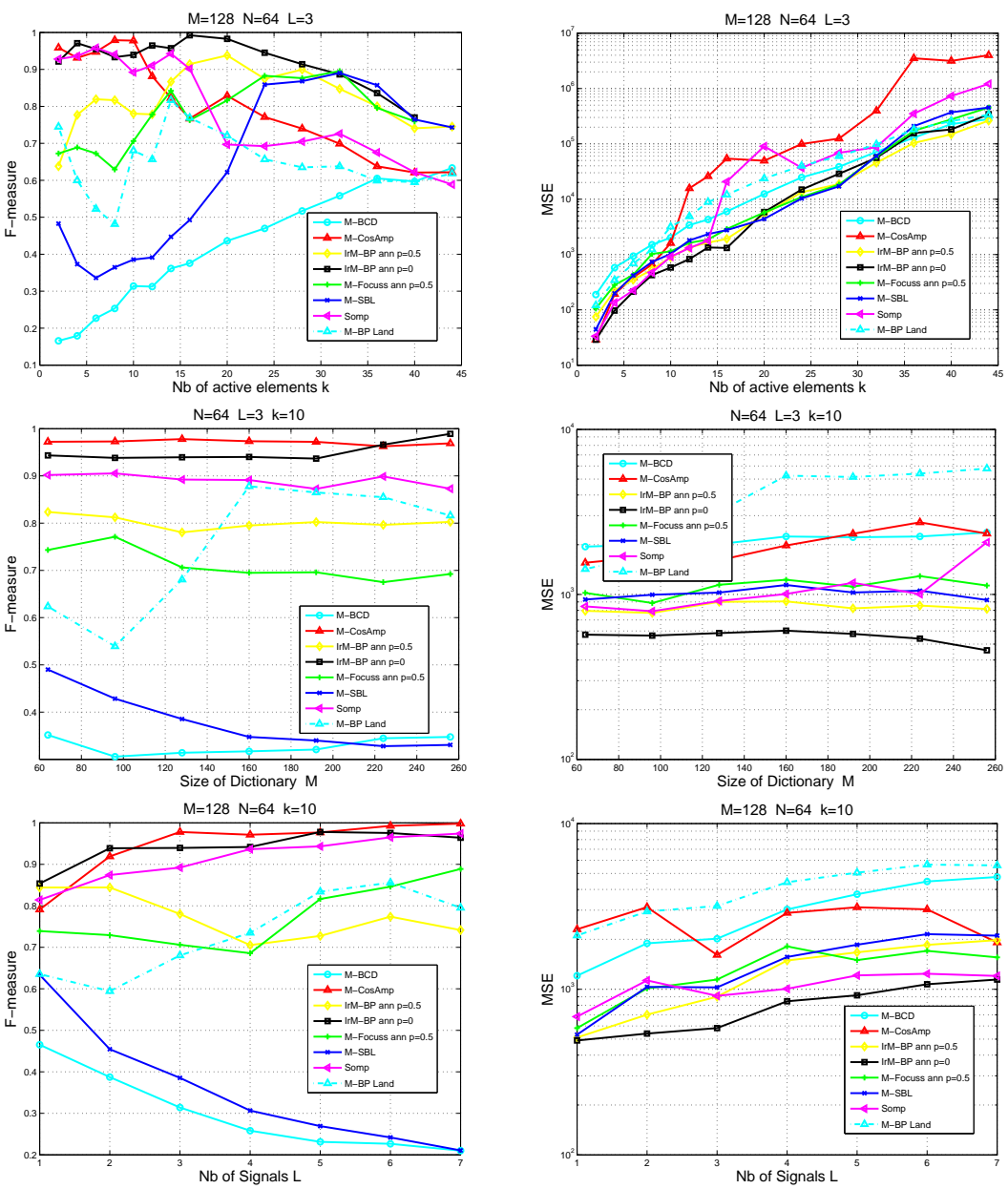

Figure 3: Results comparing performances of different simultaneous sparse algorithms. We have varied (top) the number $k$ of active elements in the dictionary. (middle) the dictionary size $M$ and (bottom) the number of signal to approximate $L$. On the left columns are given the F-measure of all methods while the average mean-square errors are on the right column. 

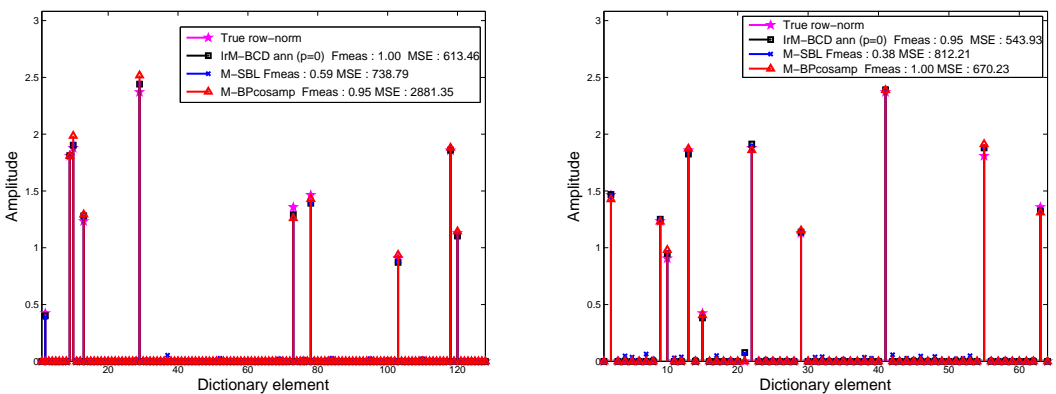

Figure 4: Examples of estimated row-norm using 3 different algorithms. left) $M=128$, $N=64, k=10$ and $L=3$. right) $M=64, N=64, k=10$ and $L=3$. Here, we want to illustrate cases where a "good" sparsity recovery does not necessary lead to low mean-square error. 